\title{
Development of triazinone-based condensing reagents for amide formation
}

Kohei Yamada, Mika Kota, Kensuke Takahashi, Hikaru Fujita, Masanori Kitamura, Munetaka Kunishima*

Faculty of Pharmaceutical Sciences, Institute of Medical, Pharmaceutical, and Health Sciences

Kanazawa University, Kakuma-machi, Kanazawa 920-1192, Japan

* Correspondence e-mail: kunisima@p.kanazawa-u.ac.jp

\section{Supporting Information}

\section{contents}

1. Amidation using 12-OiPr in the presence of diisopropylethylamine

2. Racemization test of $\mathbf{1 7 f d}$

3. Culculations

4. ${ }^{1} \mathrm{H}$ and ${ }^{13} \mathrm{C}$ NMR spectra for compounds 12-OMe, 12-OEt, 12-OiPr, 12-NMe, 1-OMe, 1-OEt, 1-OiPr, 1-NMe 
1. Amidation using 12-OiPr in the presence of diisopropylethylamine

Scheme S1

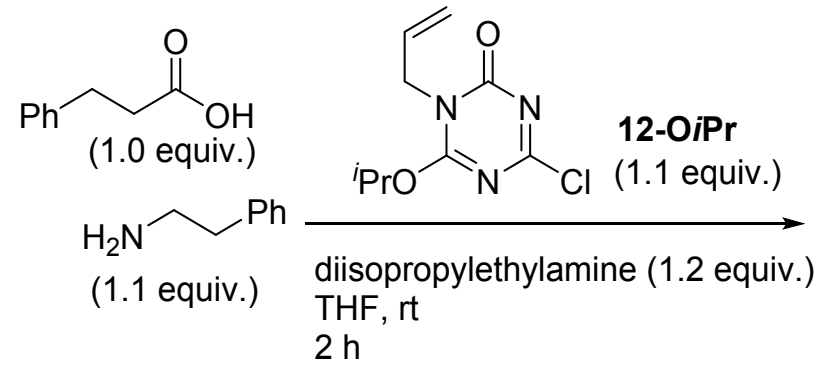<smiles>O=C(CCc1ccccc1)NCCc1ccccc1</smiles><smiles>C=CCn1c(OCCC)nc(NCCc2ccccc2)nc1=O</smiles>
$2 \mathrm{~h}$ 


\section{Racemization test of $\mathbf{1 7 f d}$}

\section{Figure S1}

a) Crude ${ }^{1} \mathrm{H}$ NMR spectrum of $\mathbf{D , L - 1 7 f d}$ in the presence of 6-methylcoumarin as an internal standard (yield: quant).

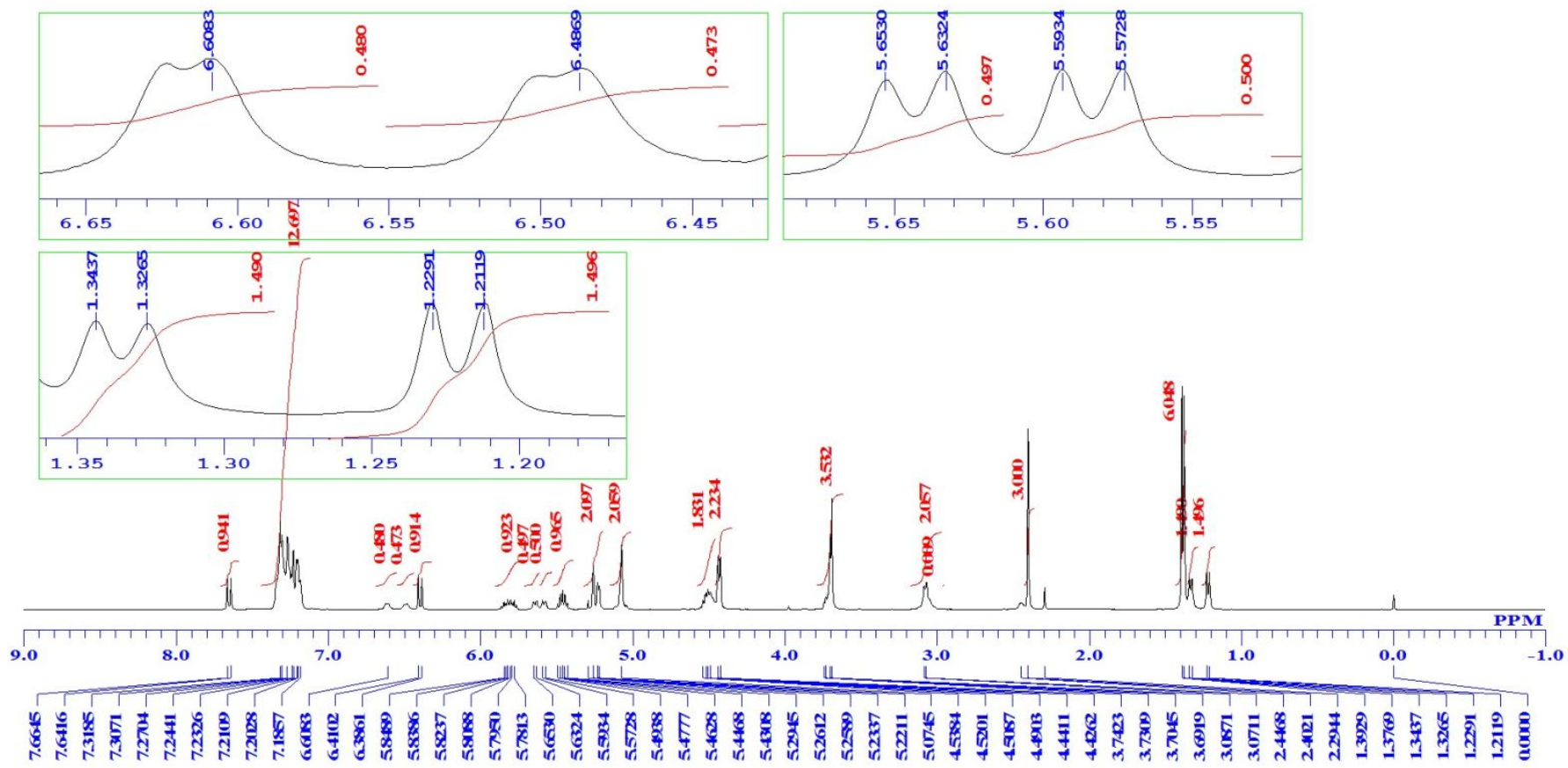

b) Crude ${ }^{1} \mathrm{H}$ NMR spectrum of $\mathbf{1 7 f d}$ in the presence of 6-methylcoumarin as an internal standard (yield: quant).

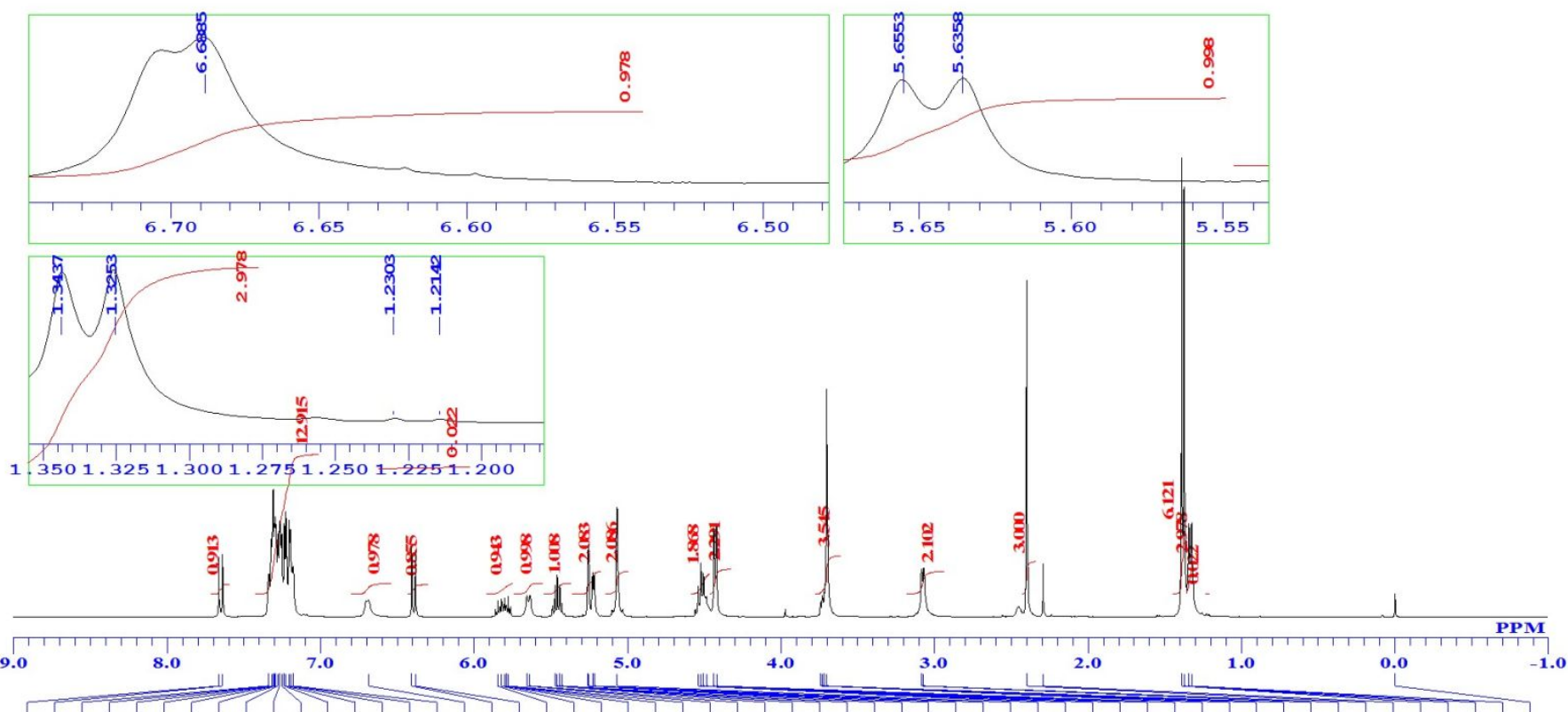

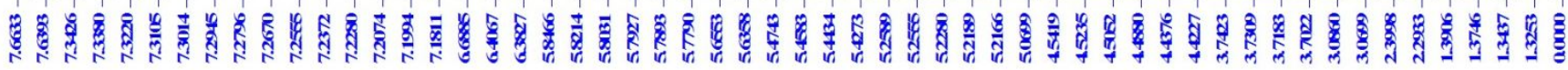

Two doublets at 1.33 and $1.22 \mathrm{ppm}$ of $\mathbf{D , L} \mathbf{L}-\mathbf{1 7 f d}$ were assigned as methyl groups of Ala side chain, indicating the presence of two diastereomers. On the other hand, in the case of $\mathbf{1 7 f d}$, the peak at $1.33 \mathrm{ppm}$ and merely trace amount of the other one at $1.22 \mathrm{ppm}(<1 \%)$ were observed. These results indicated that racemization at $\alpha$-position of phenylalanine did not occur during the reaction using 1-OiPr. 


\section{Culculations}

\subsection{Optimized Structures}

2-chloro-4,6-dimethoxy-1,3,5-triazine (CDMT)<smiles>COc1nc(Cl)nc(OC)n1</smiles>

$\begin{array}{lrrr}\mathrm{C} & -1.120826 & 0.000000 & 0.429089 \\ \mathrm{~N} & 1.191943 & 0.000000 & -0.909134 \\ \mathrm{~N} & -1.191943 & 0.000000 & -0.909134 \\ \mathrm{~N} & 0.000000 & 0.000000 & 1.158702 \\ \mathrm{C} & 1.120826 & 0.000000 & 0.429089 \\ \mathrm{C} & 0.000000 & 0.000000 & -1.486101 \\ \mathrm{O} & -2.300010 & 0.000000 & 1.041960 \\ \mathrm{O} & 2.300010 & 0.000000 & 1.041960 \\ \mathrm{C} & -2.311237 & 0.000000 & 2.477673 \\ \mathrm{C} & 2.311237 & 0.000000 & 2.477673 \\ \mathrm{Cl} & 0.000000 & 0.000000 & -3.235562 \\ \mathrm{H} & -3.365705 & 0.000000 & 2.751912 \\ \mathrm{H} & -1.814028 & 0.890110 & 2.870449 \\ \mathrm{H} & -1.814028 & -0.890110 & 2.870449 \\ \mathrm{H} & 3.365705 & 0.000000 & 2.751912 \\ \mathrm{H} & 1.814028 & 0.890110 & 2.870449 \\ \mathrm{H} & 1.814028 & -0.890110 & 2.870449\end{array}$

4-chloro-6-methoxy-1-methyl-1,3,5-triazin-2(1H)-one (3)<smiles>COc1nc(Cl)nc(=O)n1C</smiles>

$\begin{array}{lrrr}\mathrm{C} & 0.240139 & -1.732125 & 0.000000 \\ \mathrm{~N} & -0.480062 & 0.981040 & 0.000000 \\ \mathrm{~N} & -1.084883 & -1.335810 & 0.000000 \\ \mathrm{~N} & 1.211494 & -0.660365 & 0.000000 \\ \mathrm{C} & 0.784234 & 0.622711 & 0.000000 \\ \mathrm{C} & -1.335151 & -0.066113 & 0.000000 \\ \mathrm{O} & 0.618641 & -2.883575 & 0.000000 \\ \mathrm{C} & 2.640348 & -0.992746 & 0.000000 \\ \mathrm{O} & 1.754763 & 1.527995 & 0.000000 \\ \mathrm{C} & 1.358331 & 2.917530 & 0.000000 \\ \mathrm{Cl} & -3.025152 & 0.408284 & 0.000000 \\ \mathrm{H} & 2.705060 & -2.079025 & 0.000000 \\ \mathrm{H} & 3.122453 & -0.581965 & 0.890478 \\ \mathrm{H} & 3.122453 & -0.581965 & -0.890478 \\ \mathrm{H} & 0.770239 & 3.142889 & -0.891535 \\ \mathrm{H} & 0.770239 & 3.142889 & 0.891535 \\ \mathrm{H} & 2.296665 & 3.471381 & 0.000000\end{array}$


6-chloro-1,3-dimethyl-1,3,5-triazine-2,4(1H,3H)-dione (4)<smiles>Cn1c(Cl)nc(=O)n(C)c1=O</smiles>

$\begin{array}{lrcc}\mathrm{C} & 1.126440 & -1.304318 & -0.010044 \\ \mathrm{~N} & -0.254363 & -1.506734 & 0.002186 \\ \mathrm{~N} & 1.565125 & 0.042832 & 0.028503 \\ \mathrm{C} & 0.727593 & 1.140454 & 0.000868 \\ \mathrm{C} & -1.028168 & -0.491824 & 0.003682 \\ \mathrm{O} & 1.926734 & -2.213510 & -0.044154 \\ \mathrm{C} & 3.012654 & 0.289043 & 0.032937 \\ \mathrm{Cl} & -2.758725 & -0.786600 & 0.014388 \\ \mathrm{H} & 3.488681 & -0.510043 & 0.599107 \\ \mathrm{H} & 3.196752 & 1.261816 & 0.485403 \\ \mathrm{H} & 3.403146 & 0.282096 & -0.989394 \\ \mathrm{~N} & -0.658609 & 0.825789 & -0.003157 \\ \mathrm{C} & -1.608585 & 1.948099 & -0.023938 \\ \mathrm{H} & -1.013535 & 2.858681 & -0.032502 \\ \mathrm{H} & -2.242318 & 1.922908 & 0.864943 \\ \mathrm{H} & -2.231983 & 1.899040 & -0.919136 \\ \mathrm{O} & 1.117378 & 2.292982 & -0.014193\end{array}$

\subsection{Enegies}

2-chloro-4,6-dimethoxy-1,3,5-triazine (CDMT)<smiles>COc1nc(Cl)nc(OC)n1</smiles>

SCF total energy: $\quad-969.0522764$ (a.u.)

4-chloro-6-methoxy-1-methyl-1,3,5-triazin-2(1H)-one (3)<smiles>COc1nc(Cl)nc(=O)n1C</smiles>

SCF total energy: $\quad-969.0563967$ (a.u.)

6-chloro-1,3-dimethyl-1,3,5-triazine-2,4(1H,3H)-dione (4)<smiles>Cn1c(Cl)nc(=O)n(C)c1=O</smiles>

SCF total energy: $\quad-969.0768515$ (a.u.) 
4. ${ }^{1} \mathrm{H}$ and ${ }^{13} \mathrm{C}$ NMR spectra for compounds $12-\mathrm{OMe}, 12-\mathrm{OEt}, 12-\mathrm{O} i \mathrm{Pr}, 12-\mathrm{NMe}_{2}$, 1-OMe, 1-OEt, 1-OiPr, 1-NMe ${ }^{1}$ H NMR: 1-Allyl-4-chloro-6-methoxy-1,3,5-triazin-2(1H)-one (12-OMe)
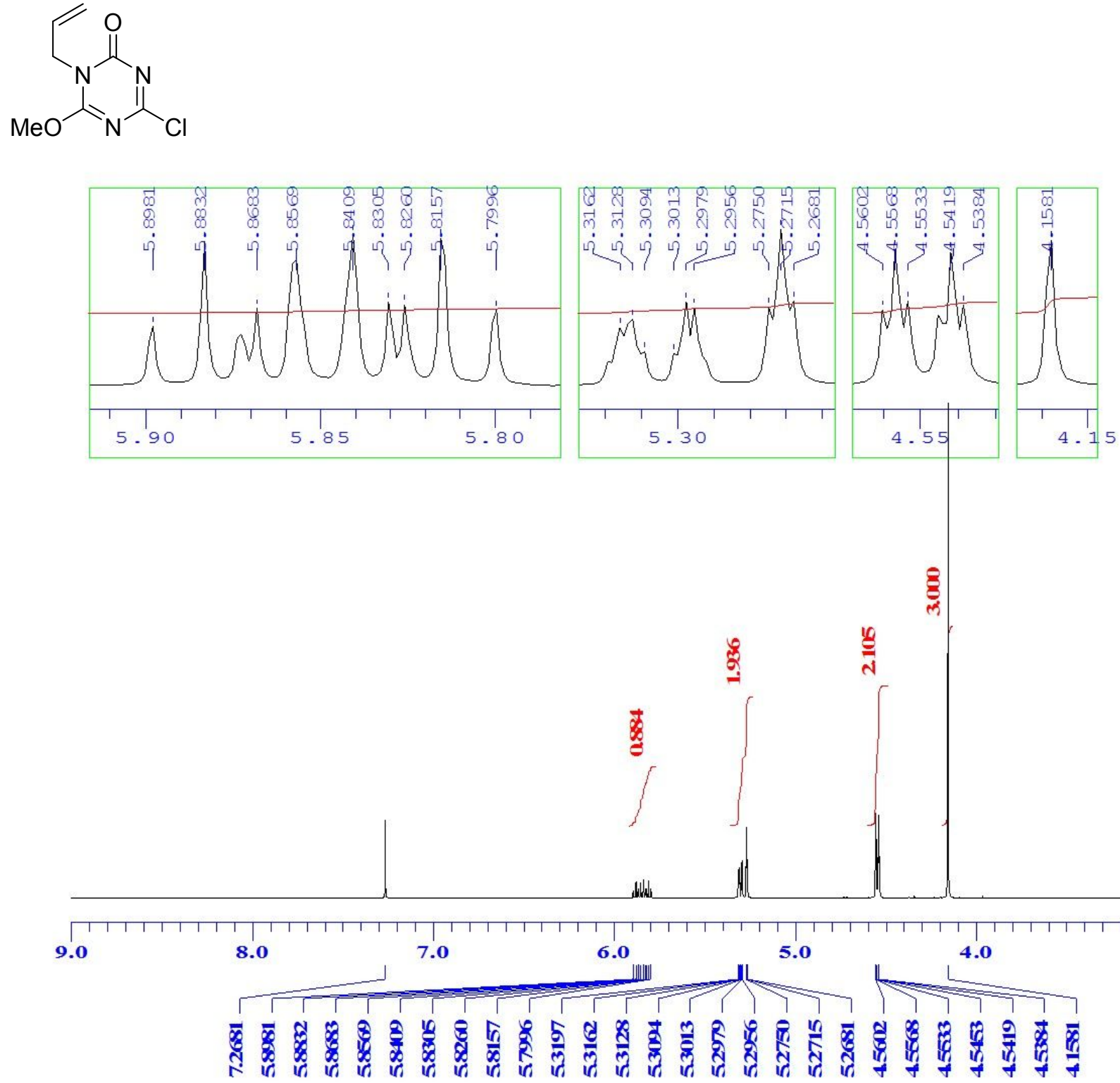
${ }^{13}$ C NMR: 1-Allyl-4-chloro-6-methoxy-1,3,5-triazin-2(1H)-one (12-OMe)
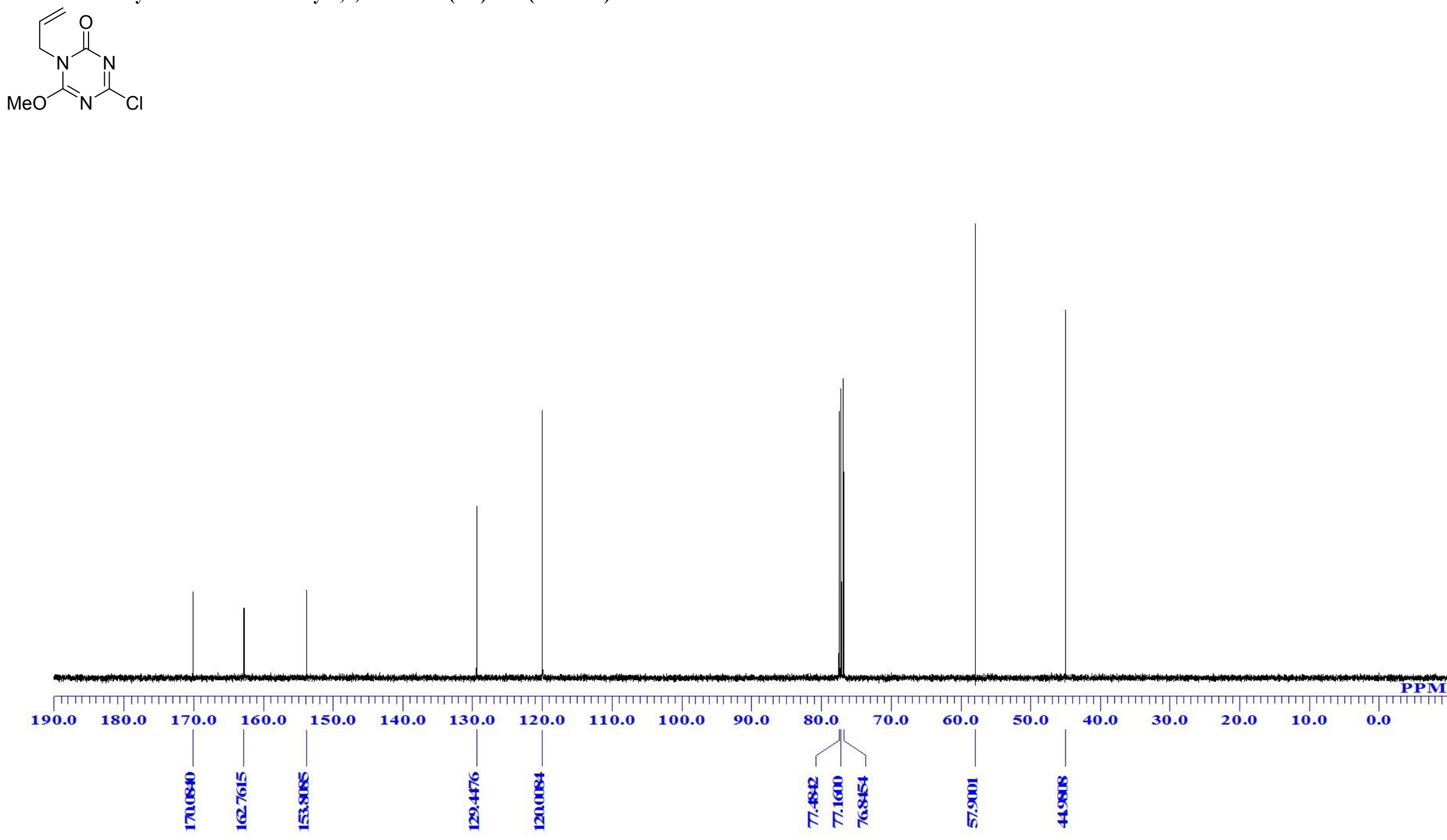
${ }^{1}$ H NMR: 1-Allyl-4-chloro-6-ethoxy-1,3,5-triazin-2(1H)-one (12-OEt)

$\overbrace{\mathrm{Cl}}$
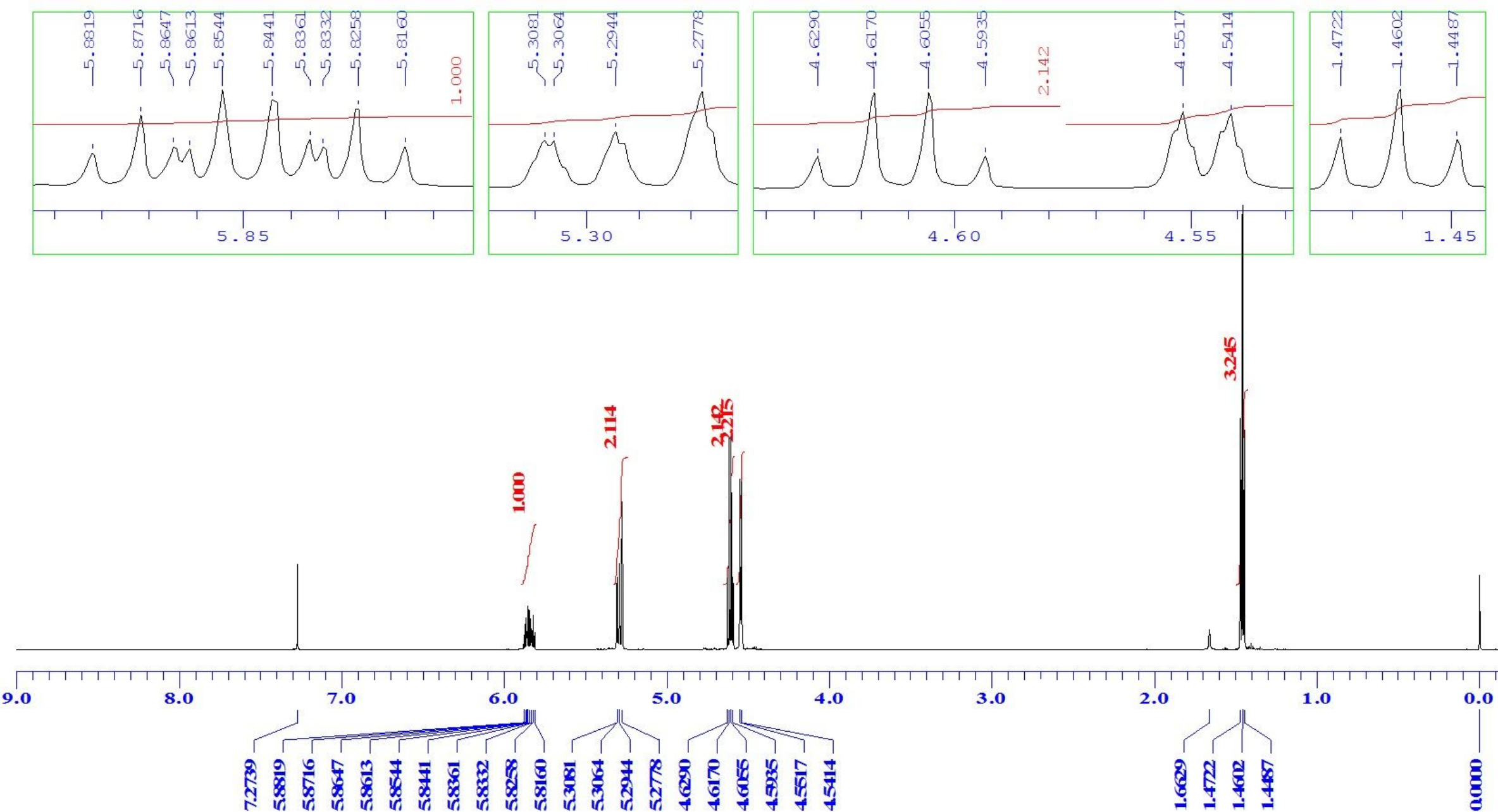
${ }^{13}$ C NMR: 1-Allyl-4-chloro-6-ethoxy-1,3,5-triazin-2(1 H)-one (12-OEt)
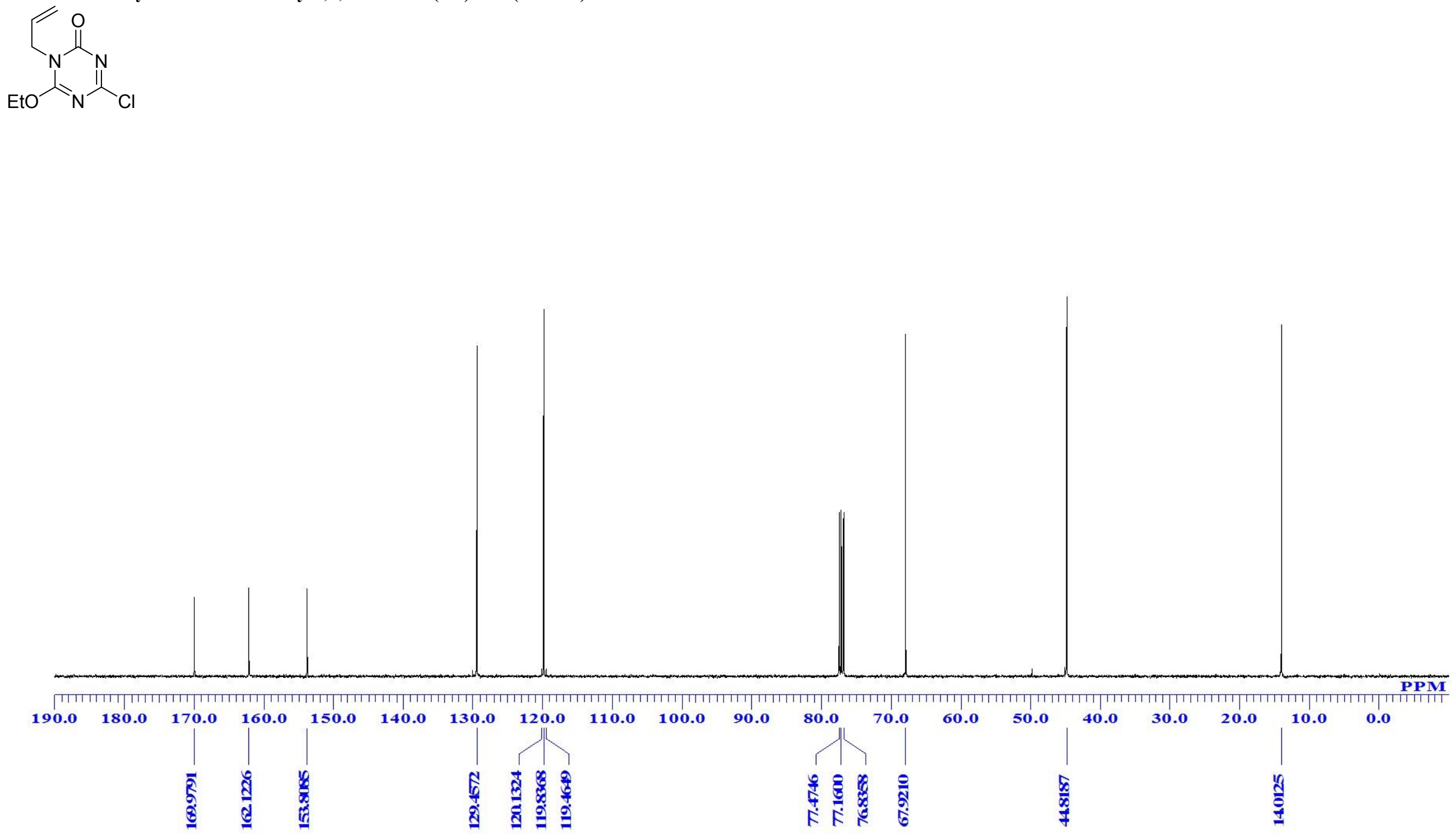
${ }^{1}$ H NMR: 1-Allyl-4-chloro-6-isopropoxy-1,3,5-triazin-2(1H)-one (12-OiPr)
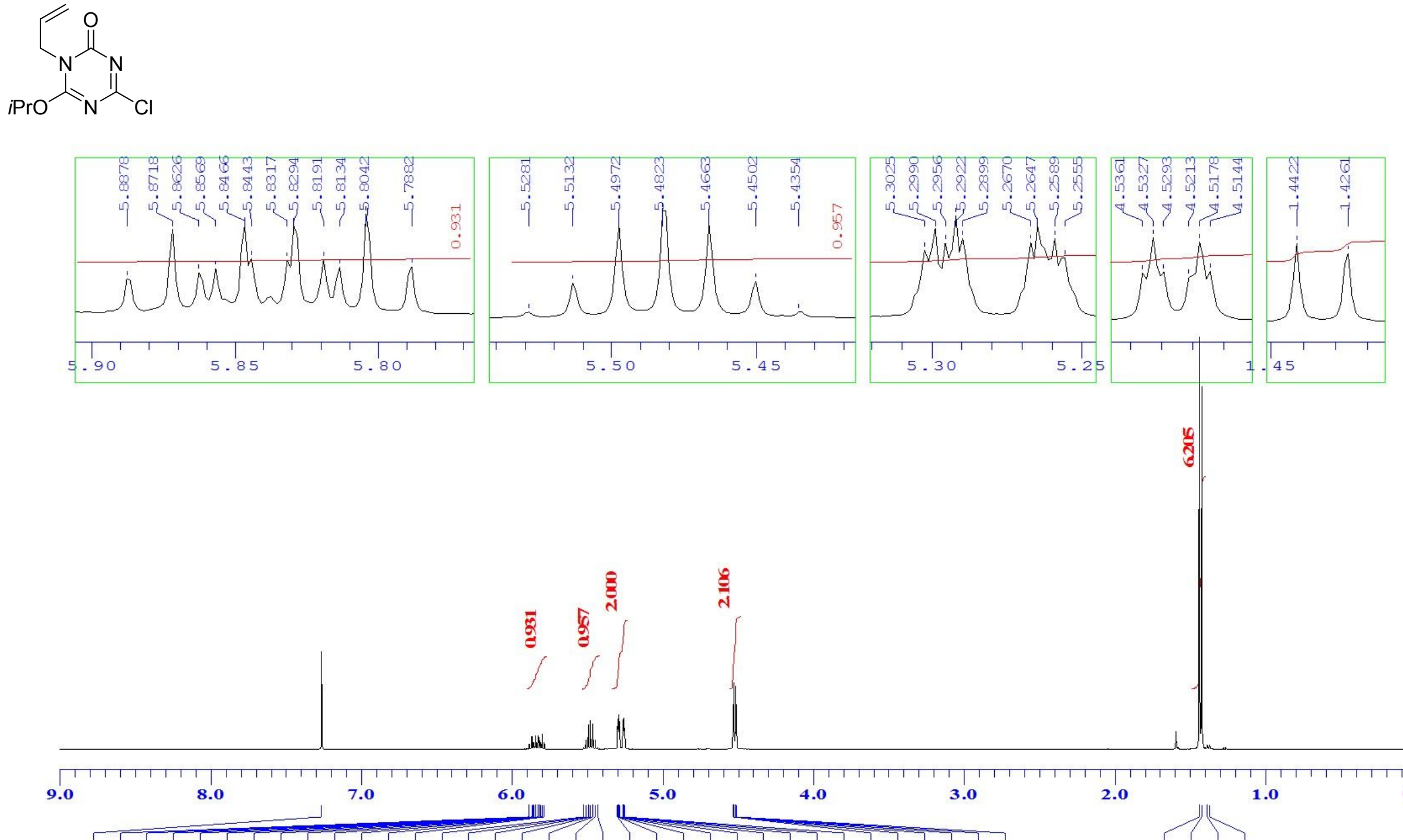

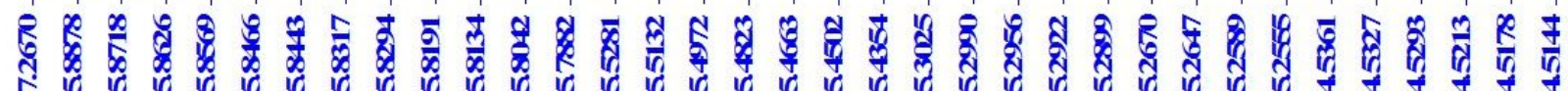


${ }^{13}$ C NMR: 1-Allyl-4-chloro-6-isopropoxy-1,3,5-triazin-2(1H)-one (12-OiPr)
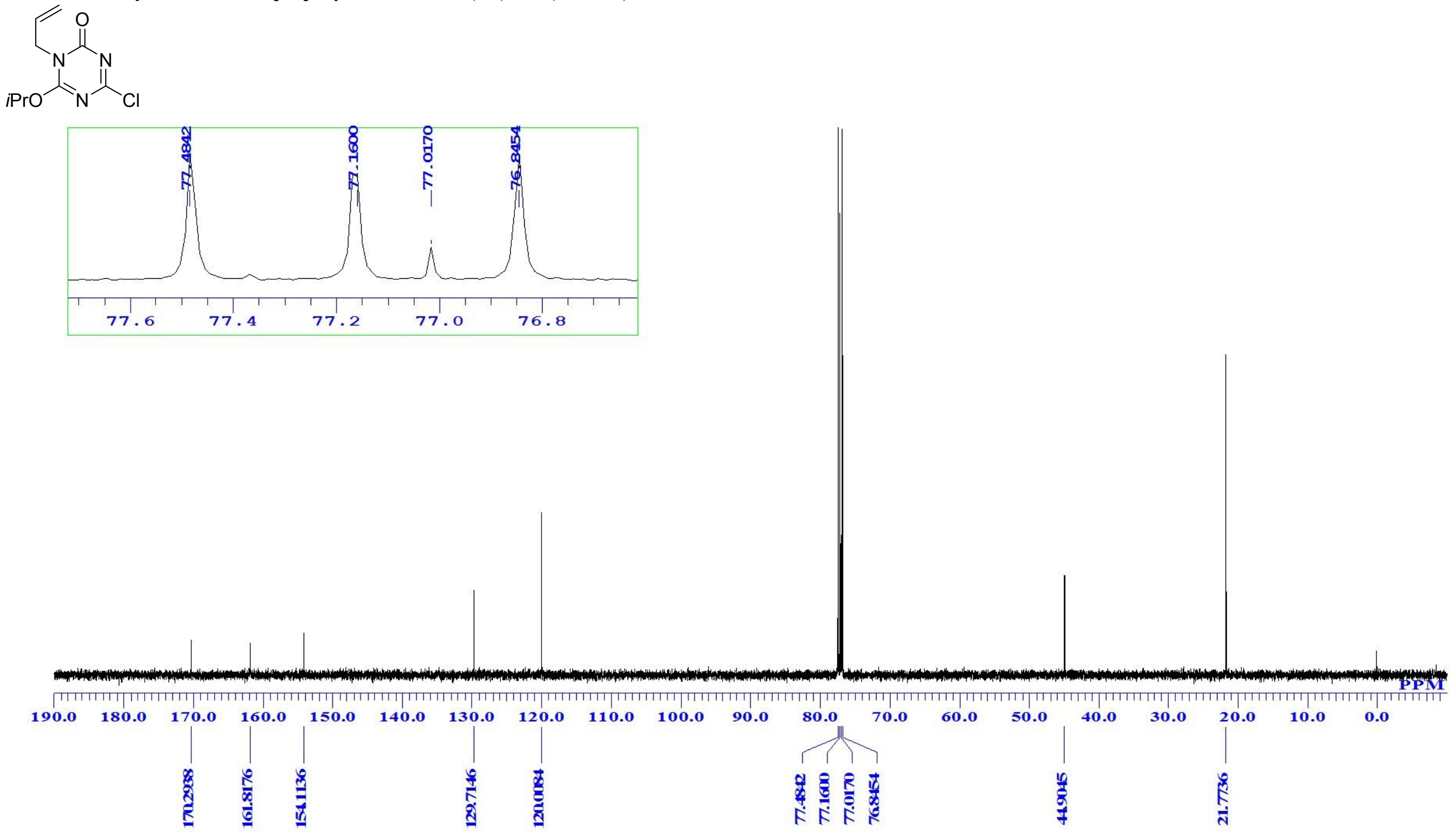
${ }^{1}$ H NMR: 1-Allyl-4-chloro-6-(dimethylamino)-1,3,5-triazin-2(1H)-one (12-NMe $)$

$\mathrm{Me}_{2}{ }_{\mathrm{N}}$
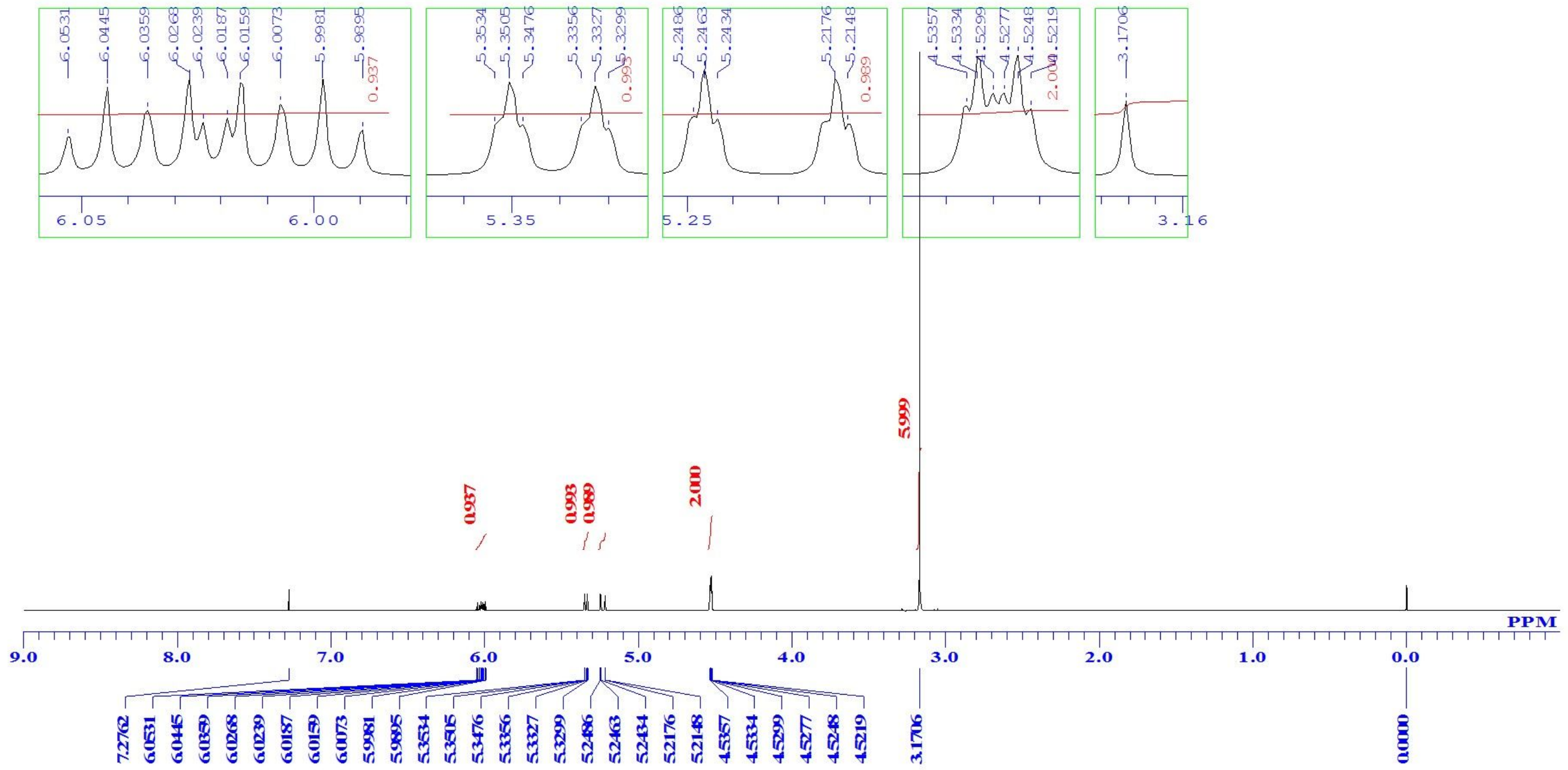
${ }^{13} \mathrm{C}$ NMR: 1-Allyl-4-chloro-6-(dimethylamino)-1,3,5-triazin-2(1H)-one (12-NMe $)$
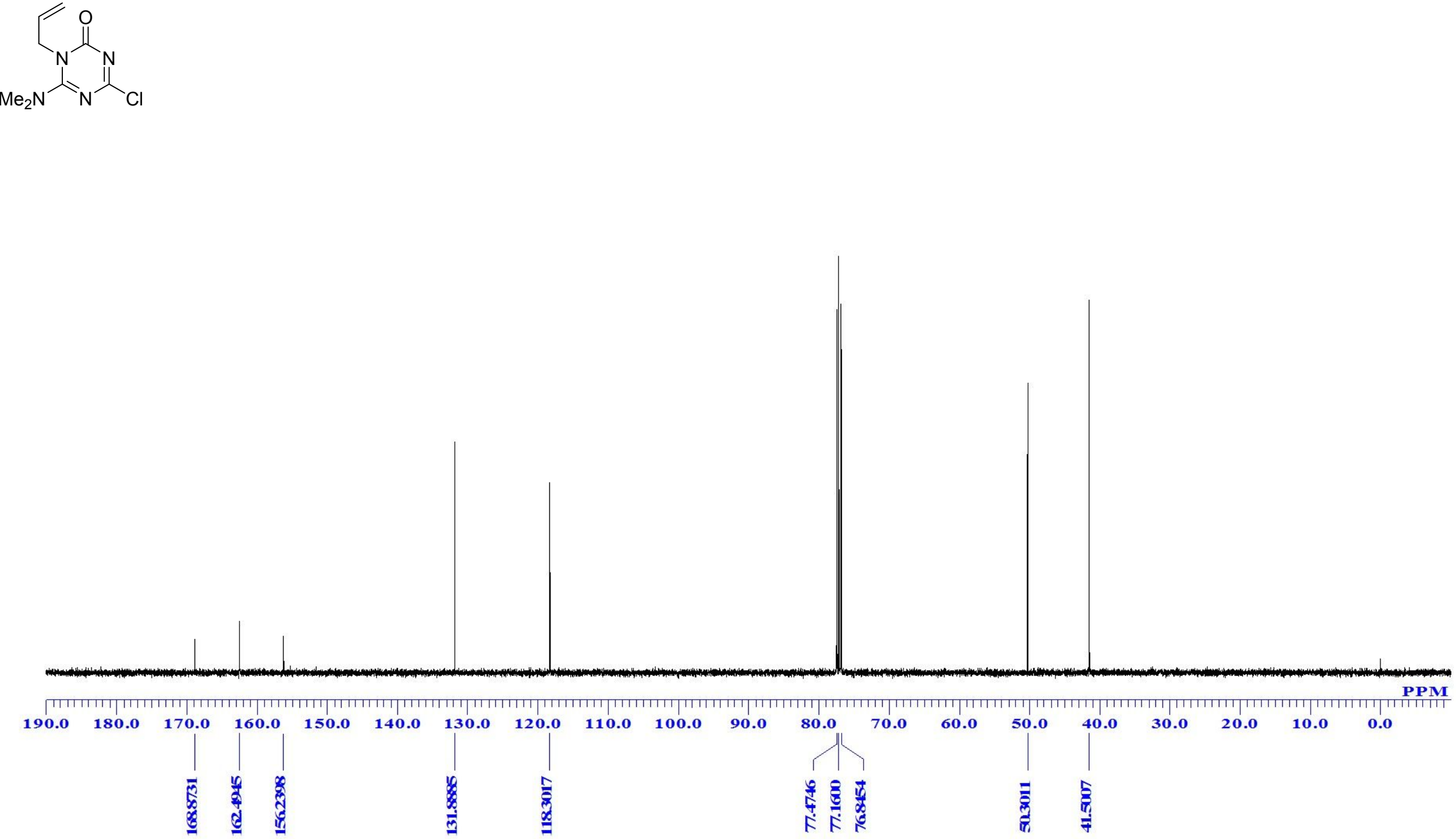
${ }^{1}$ H NMR: 4-(5-Allyl-6-methoxy-4-oxo-4,5-dihydro-1,3,5-triazin-2-yl)-4-methylmorpholinium chloride (1-OMe)
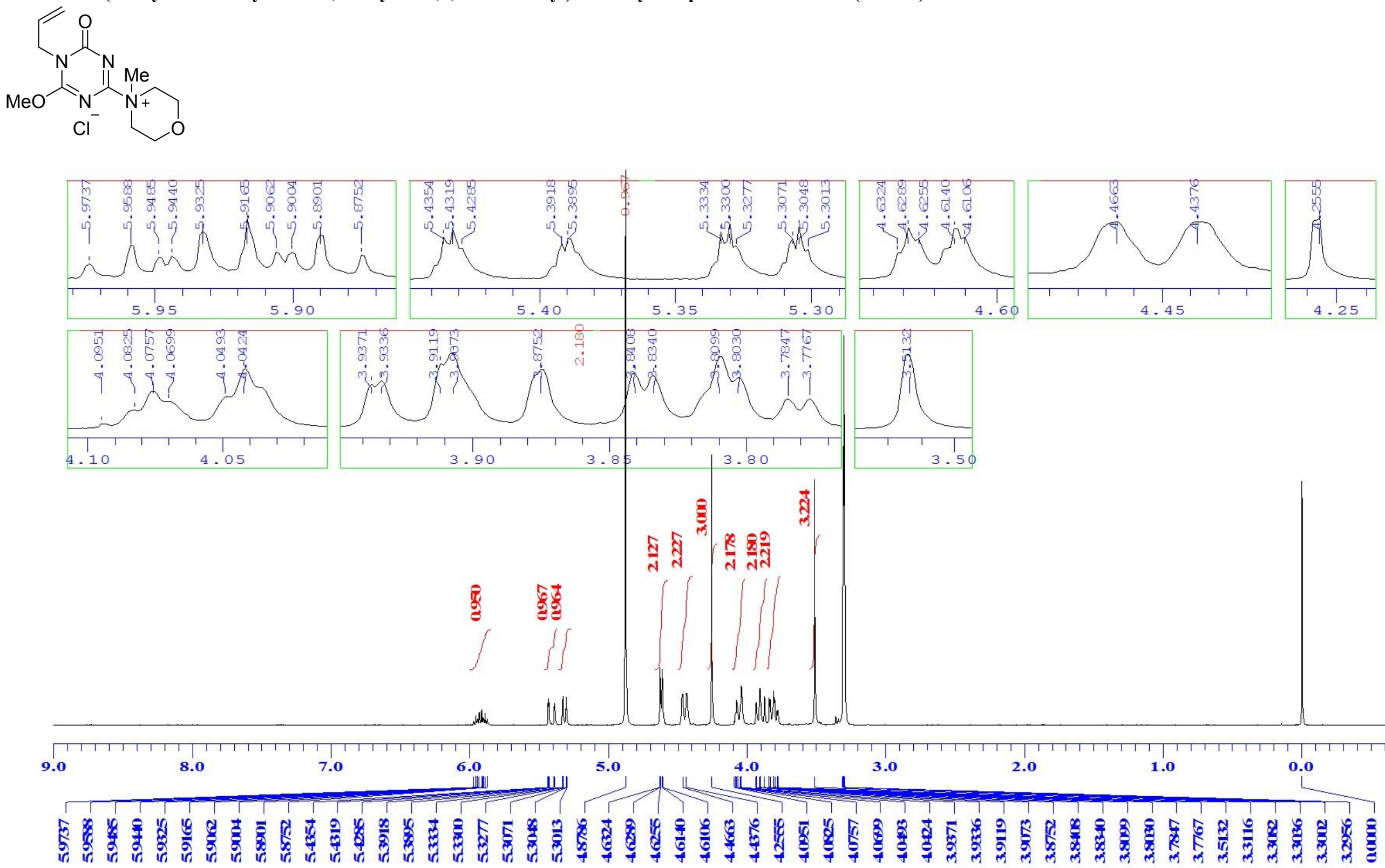
${ }^{13}$ C NMR: 4-(5-Allyl-6-methoxy-4-oxo-4,5-dihydro-1,3,5-triazin-2-yl)-4-methylmorpholinium chloride (1-OMe)
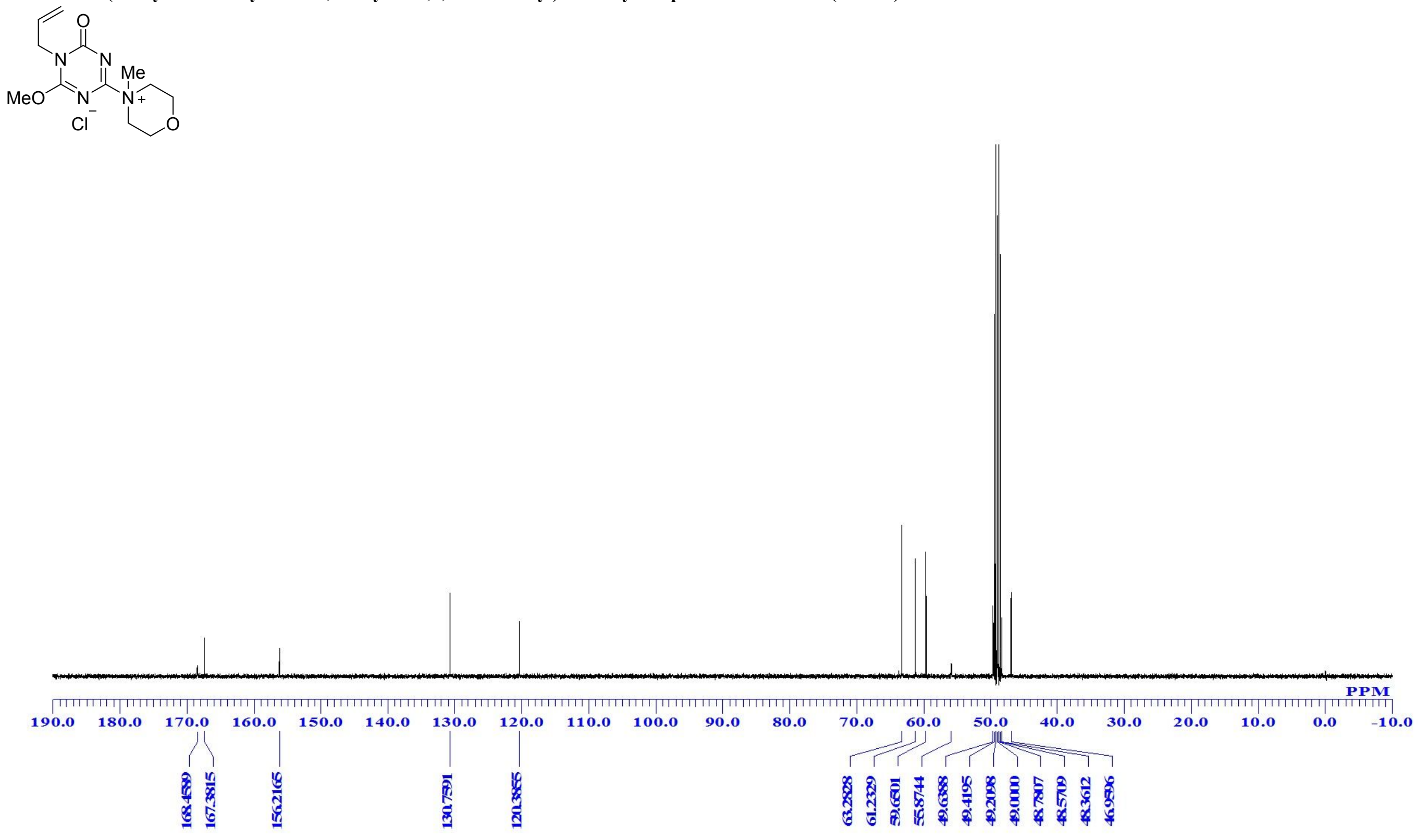
${ }^{1}$ H NMR: 4-(5-Allyl-6-ethoxy-4-oxo-4,5-dihydro-1,3,5-triazin-2-yl)-4-methylmorpholinium chloride (1-OEt)
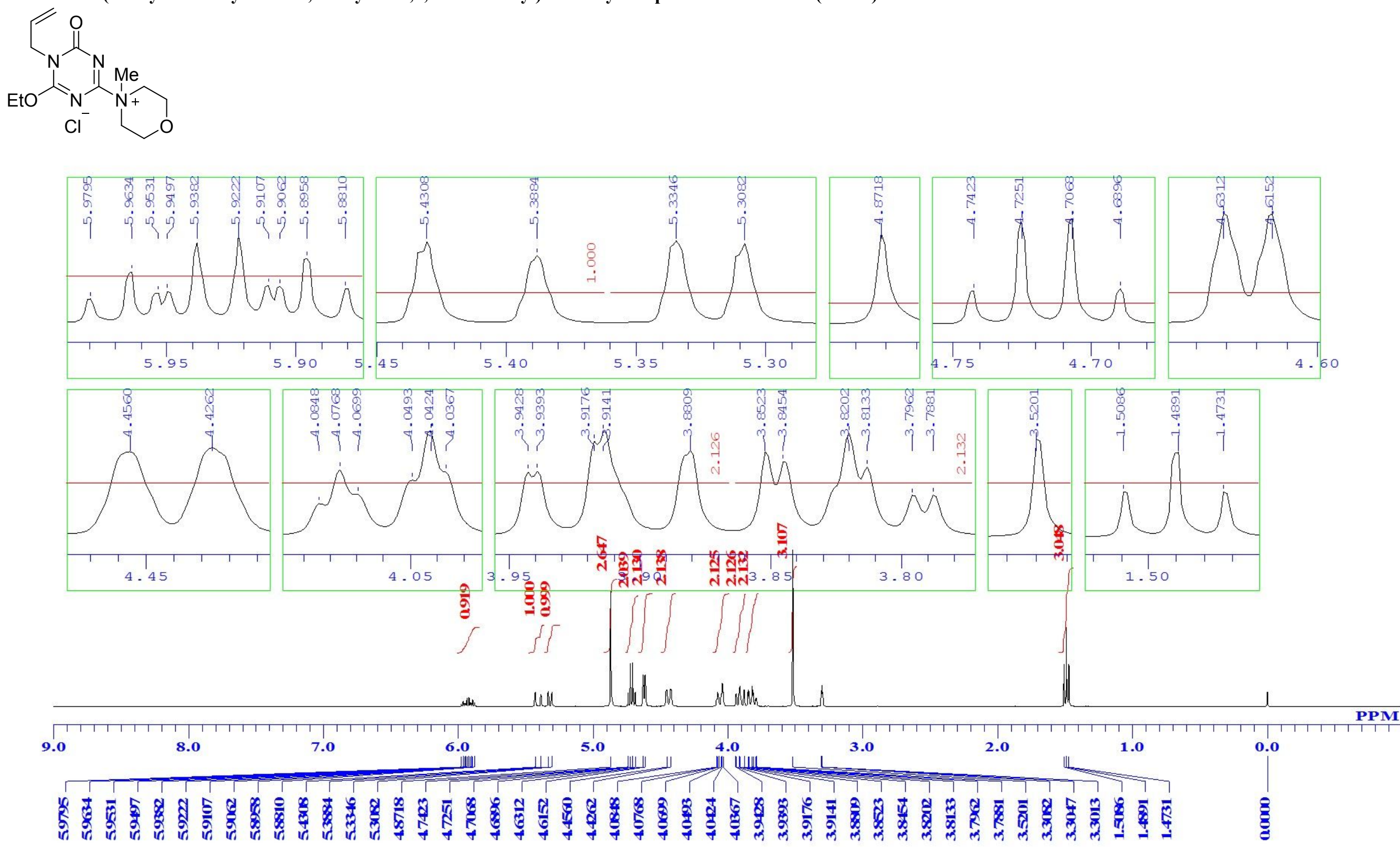
${ }^{13}$ C NMR: 4-(5-Allyl-6-ethoxy-4-oxo-4,5-dihydro-1,3,5-triazin-2-yl)-4-methylmorpholinium chloride (1-OEt)

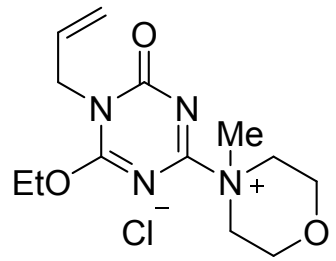

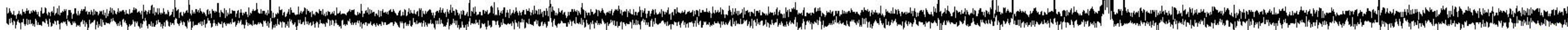

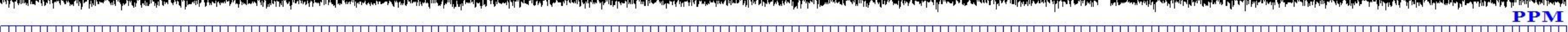

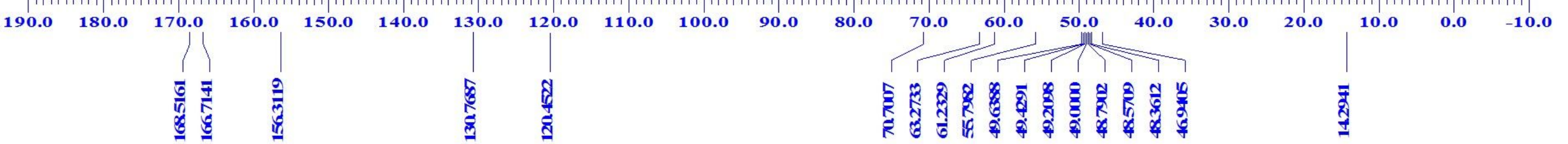


${ }^{1}$ H NMR: 4-(5-Allyl-6-isopropoxy-4-oxo-4,5-dihydro-1,3,5-triazin-2-yl)-4-methylmorpholinium chloride (1-OiPr)
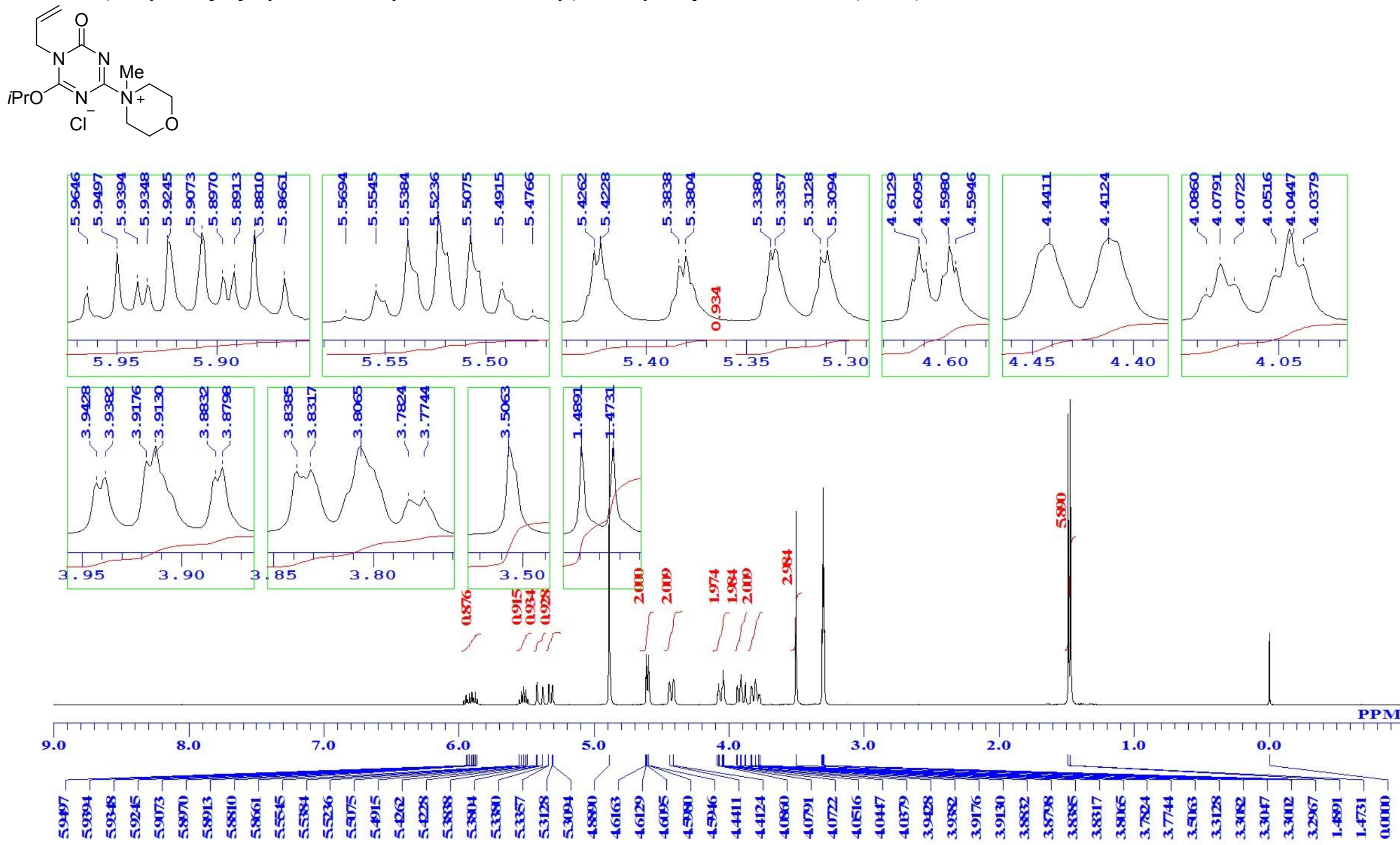
${ }^{13}$ C NMR: 4-(5-Allyl-6-isopropoxy-4-oxo-4,5-dihydro-1,3,5-triazin-2-yl)-4-methylmorpholinium chloride (1-OiPr)

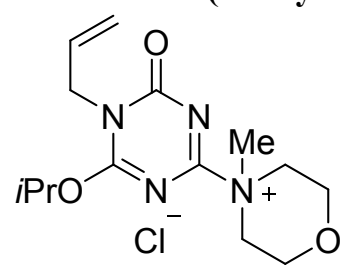

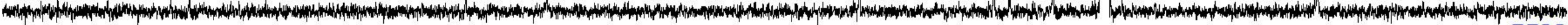

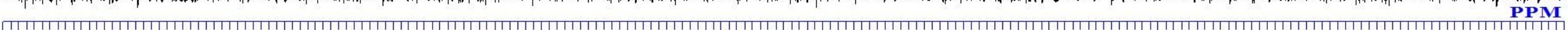

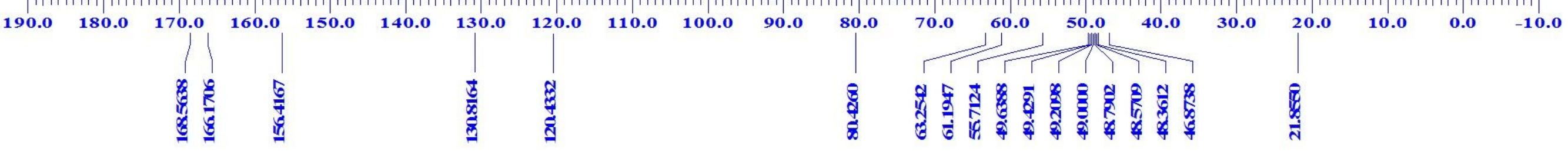


${ }^{1}$ H NMR: 4-(5-Allyl-6-(dimethylamino)-4-oxo-4,5-dihydro-1,3,5-triazin-2-yl)-4-methylmorpholin-4-ium chloride (1-NMe 2 )
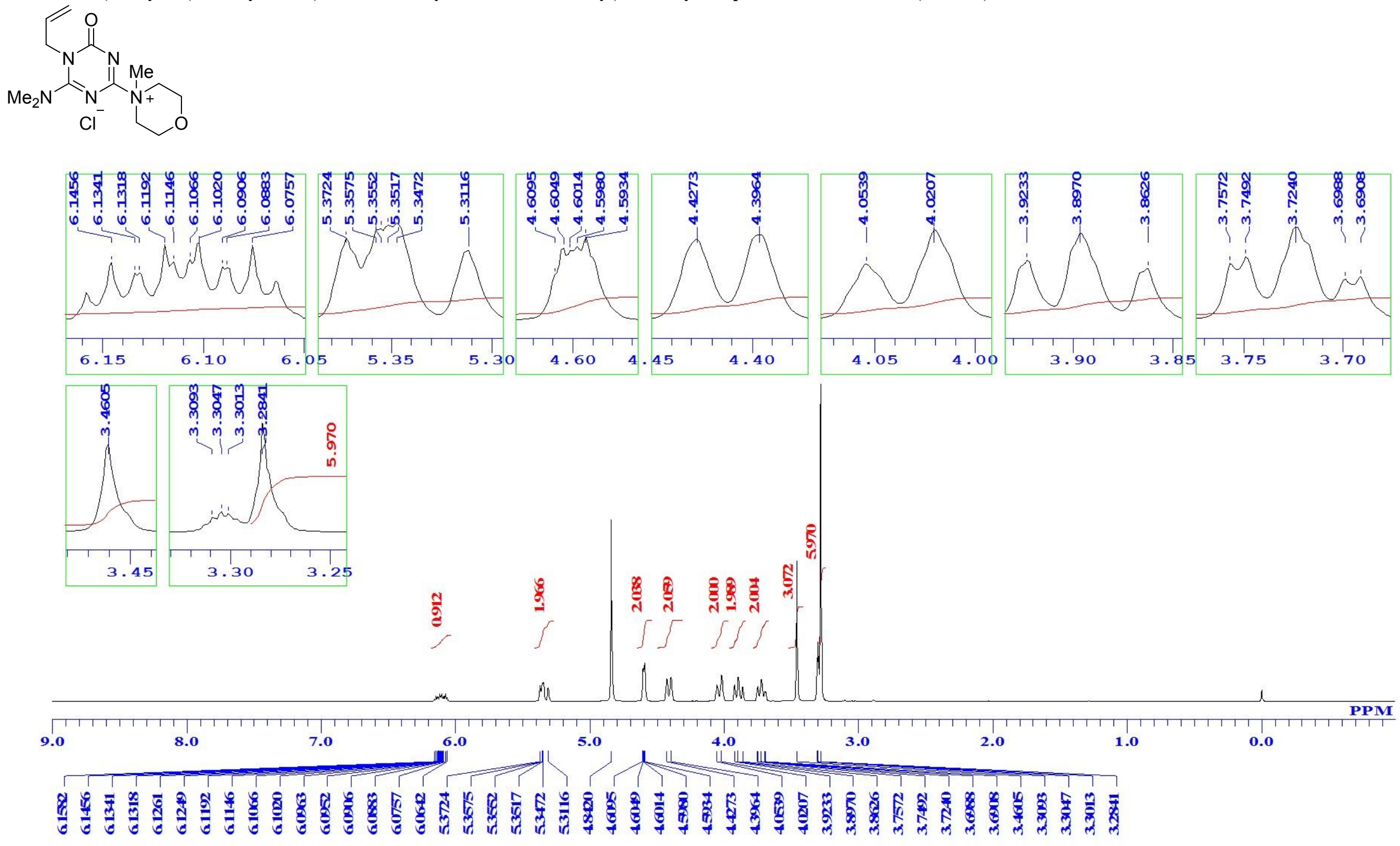
${ }^{13}$ C NMR:4-(5-Allyl-6-(dimethylamino)-4-oxo-4,5-dihydro-1,3,5-triazin-2-yl)-4-methylmorpholin-4-ium chloride (1-NMe 2 )
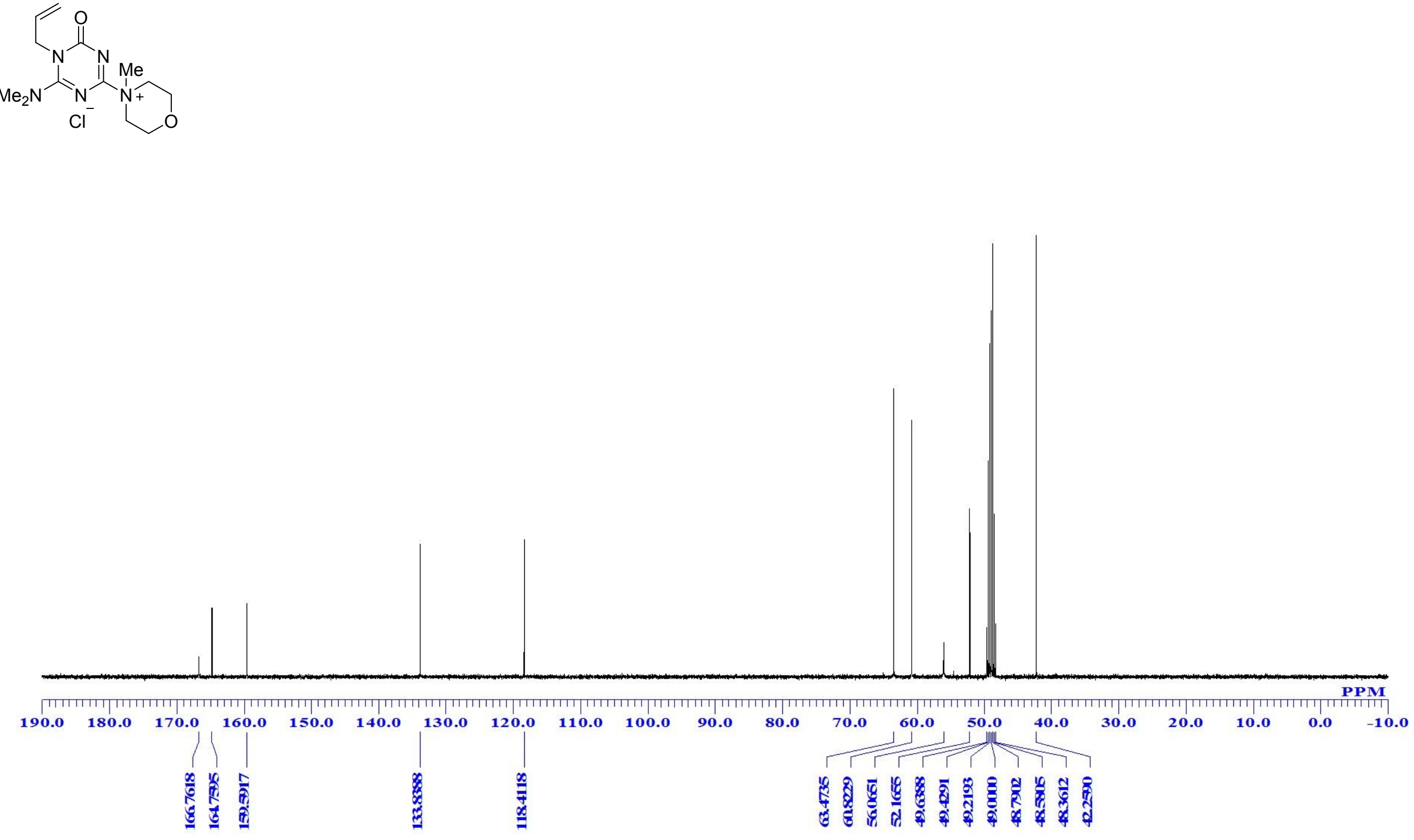
$\overbrace{\mathrm{H}}^{\mathrm{O}} \sim \mathrm{Ph}$
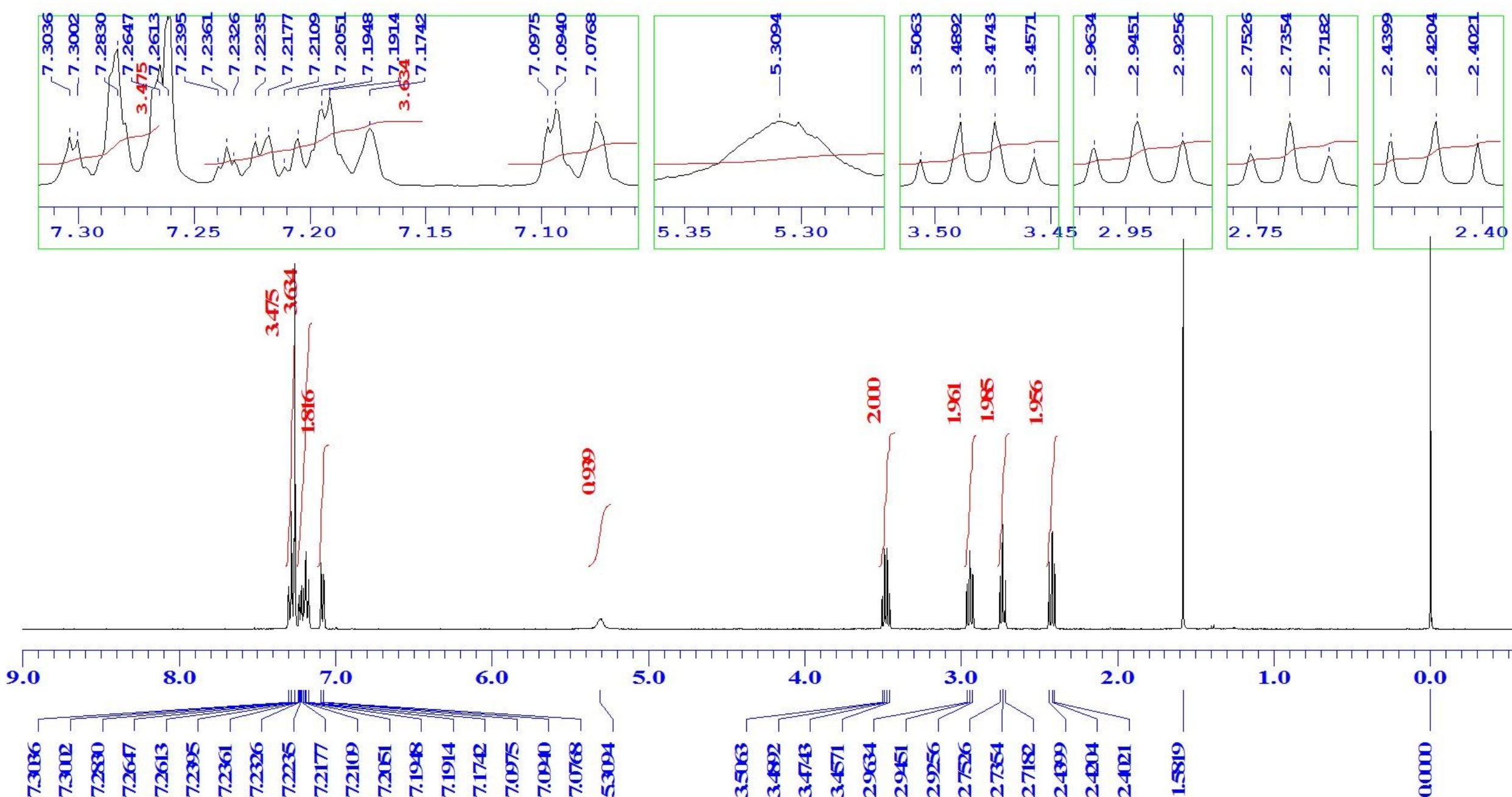

$-1.0$

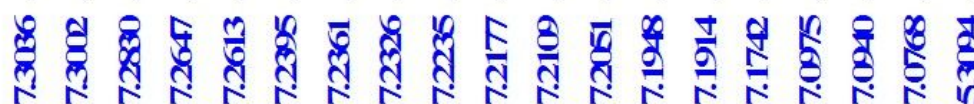

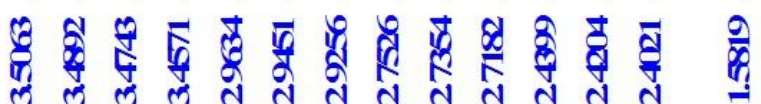

8 
${ }^{1}$ H NMR: $N, N$-Diethyl- 3-phenylpropanamide (17ab)

$\sim \sim_{M e}^{\mathrm{O}} \overbrace{\mathrm{Me}}$
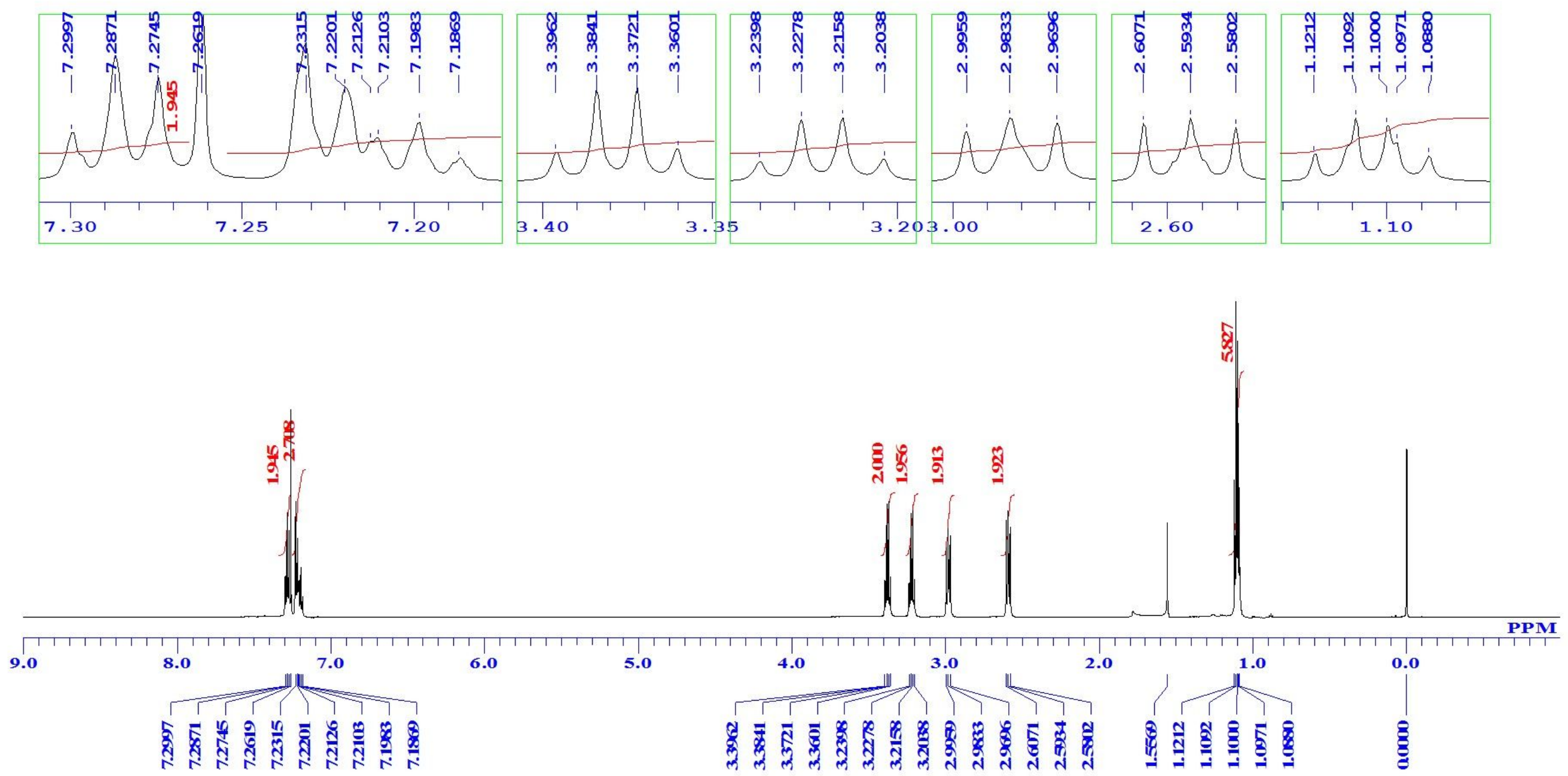
${ }^{1}$ H NMR: $N, N$-Dibenzyl- 3-phenylpropanamide (17ac)

$\overbrace{L_{\mathrm{Ph}}}^{\mathrm{O}} \widehat{\mathrm{Ph}}_{\mathrm{Ph}}$
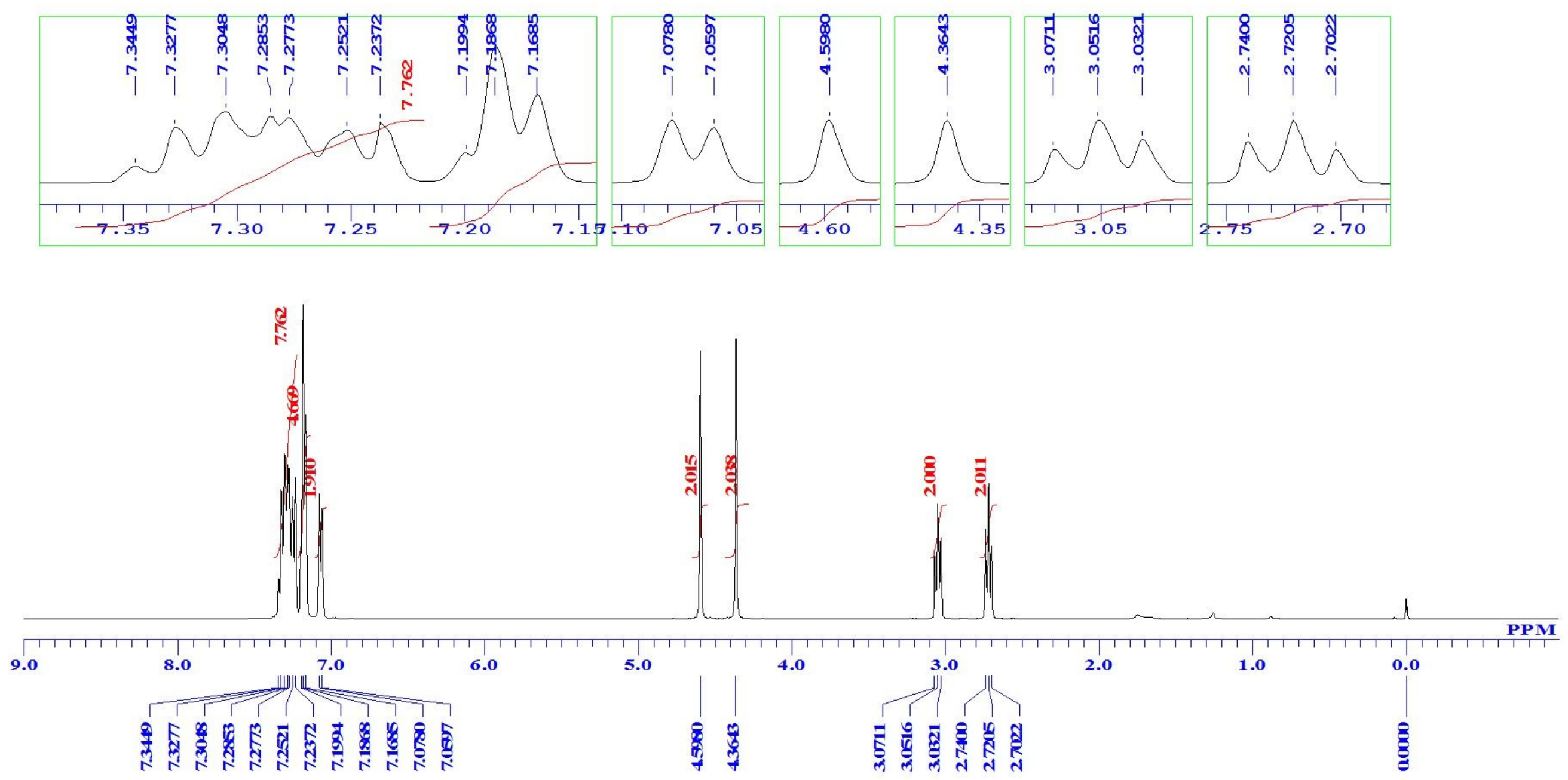
${ }^{1}$ H NMR: $N$-Phenethylpivalamide (17ba)

${ }_{M e}^{M e}$
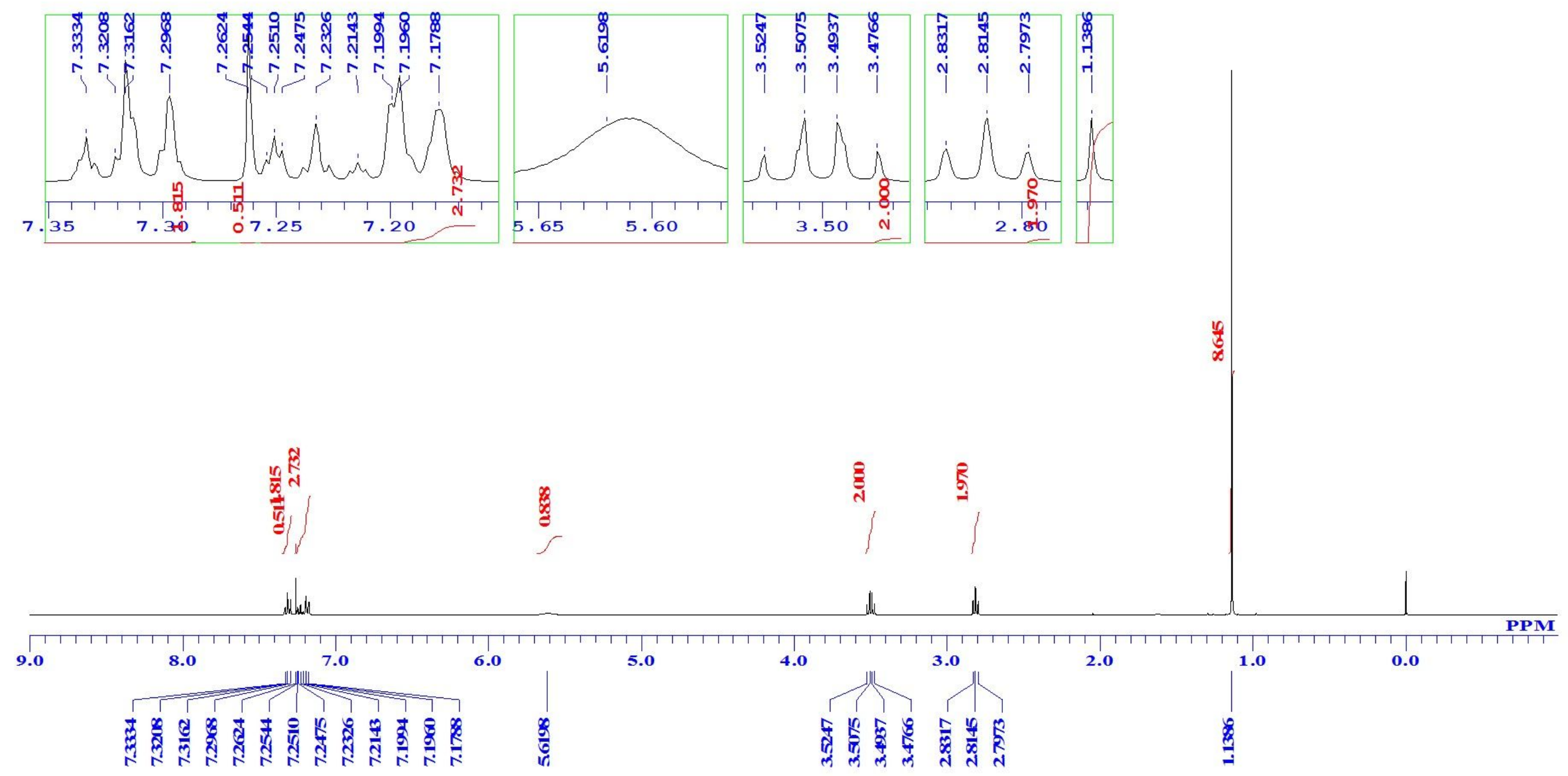
${ }^{1}$ H NMR: $N$-Phenethylbenzamide $(17 \mathrm{ca})$

$\overbrace{\mathrm{H}}^{\mathrm{O}} \sim_{\mathrm{Nh}}^{\mathrm{P}}$

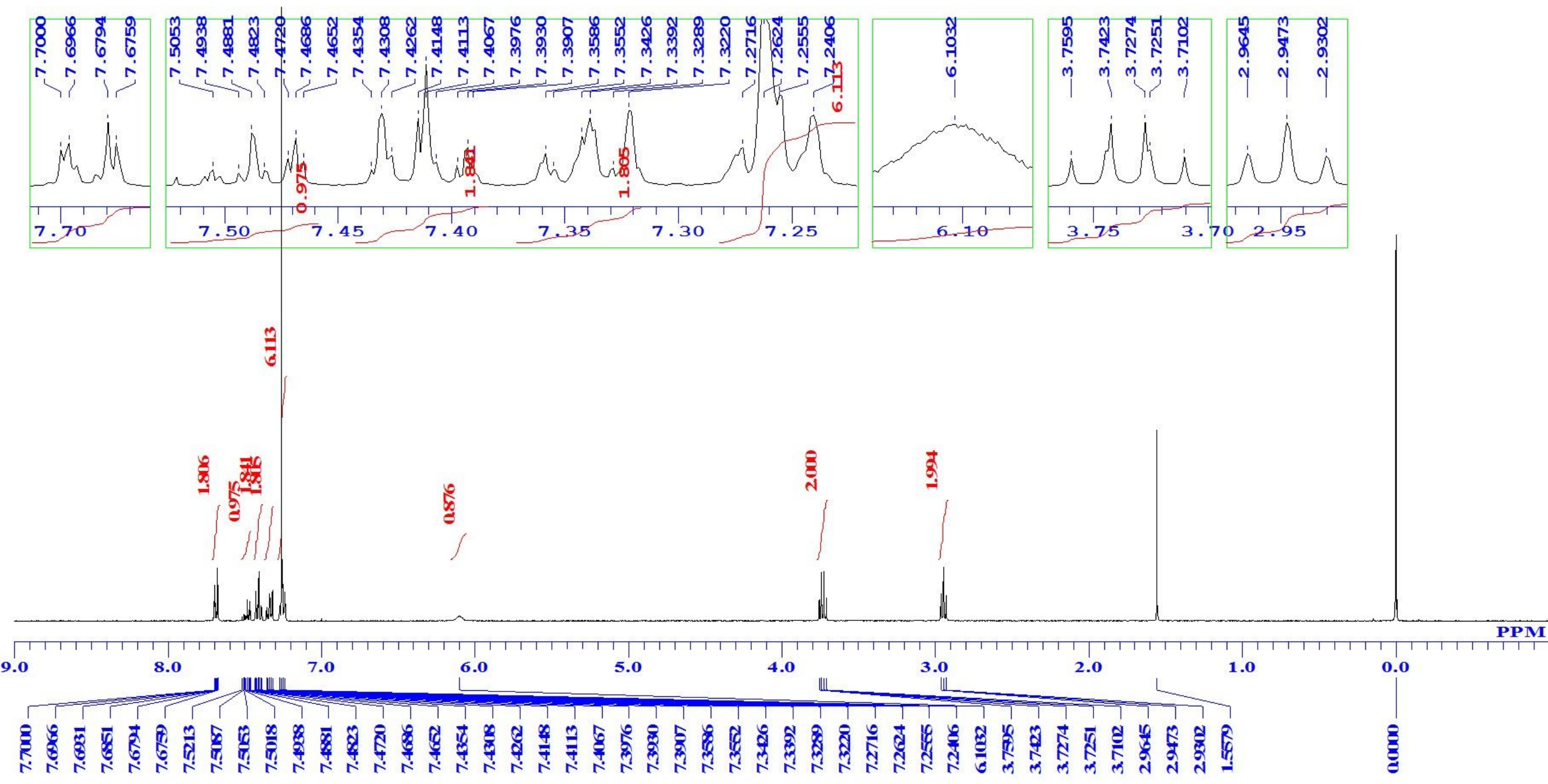


${ }^{1}$ H NMR: $N$-Phenethyl-4-methoxybenzamide (17da)

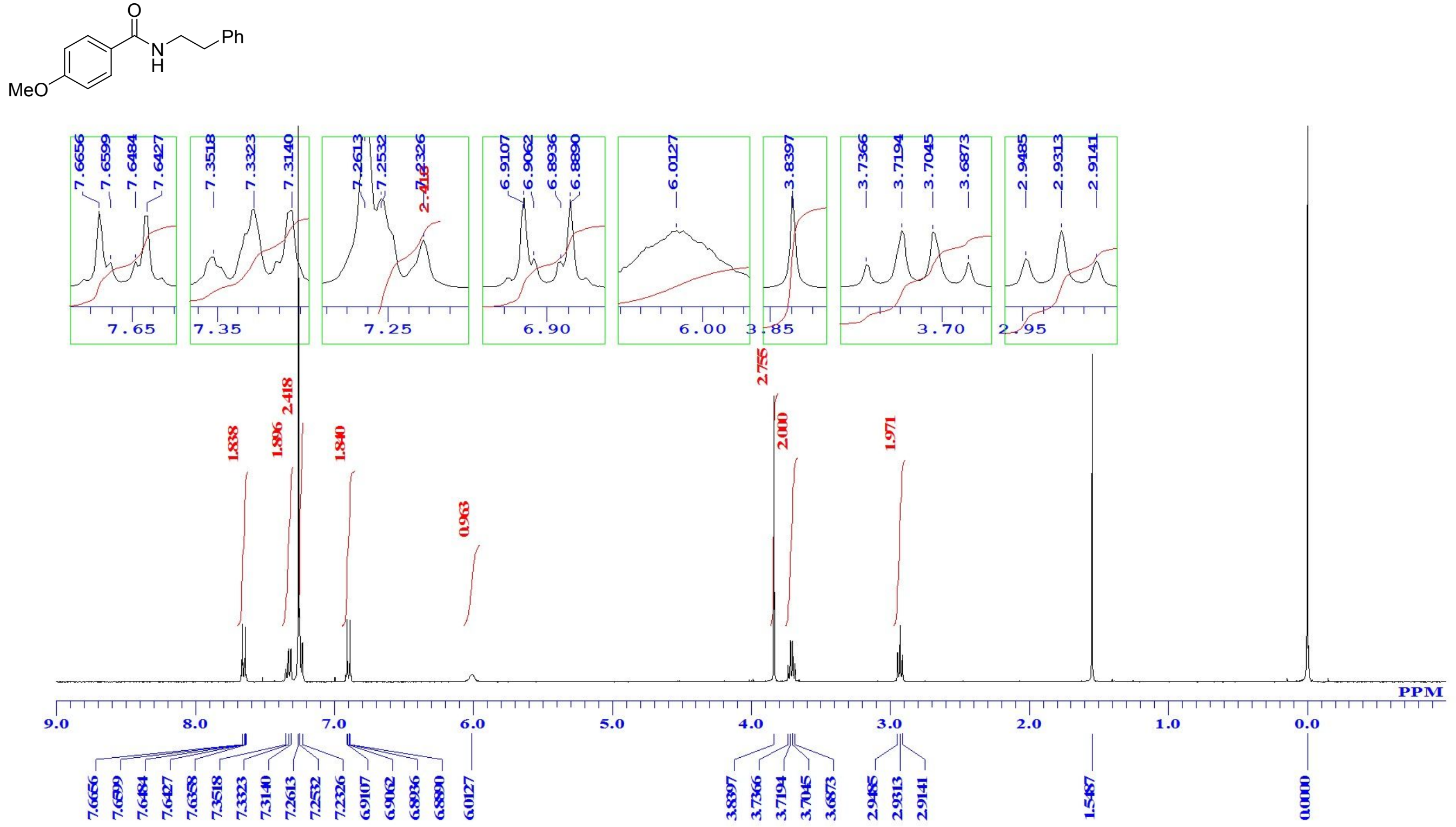


${ }^{1}$ H NMR: $N$-Phenethyl-4-nitrobenzamide (17ea)

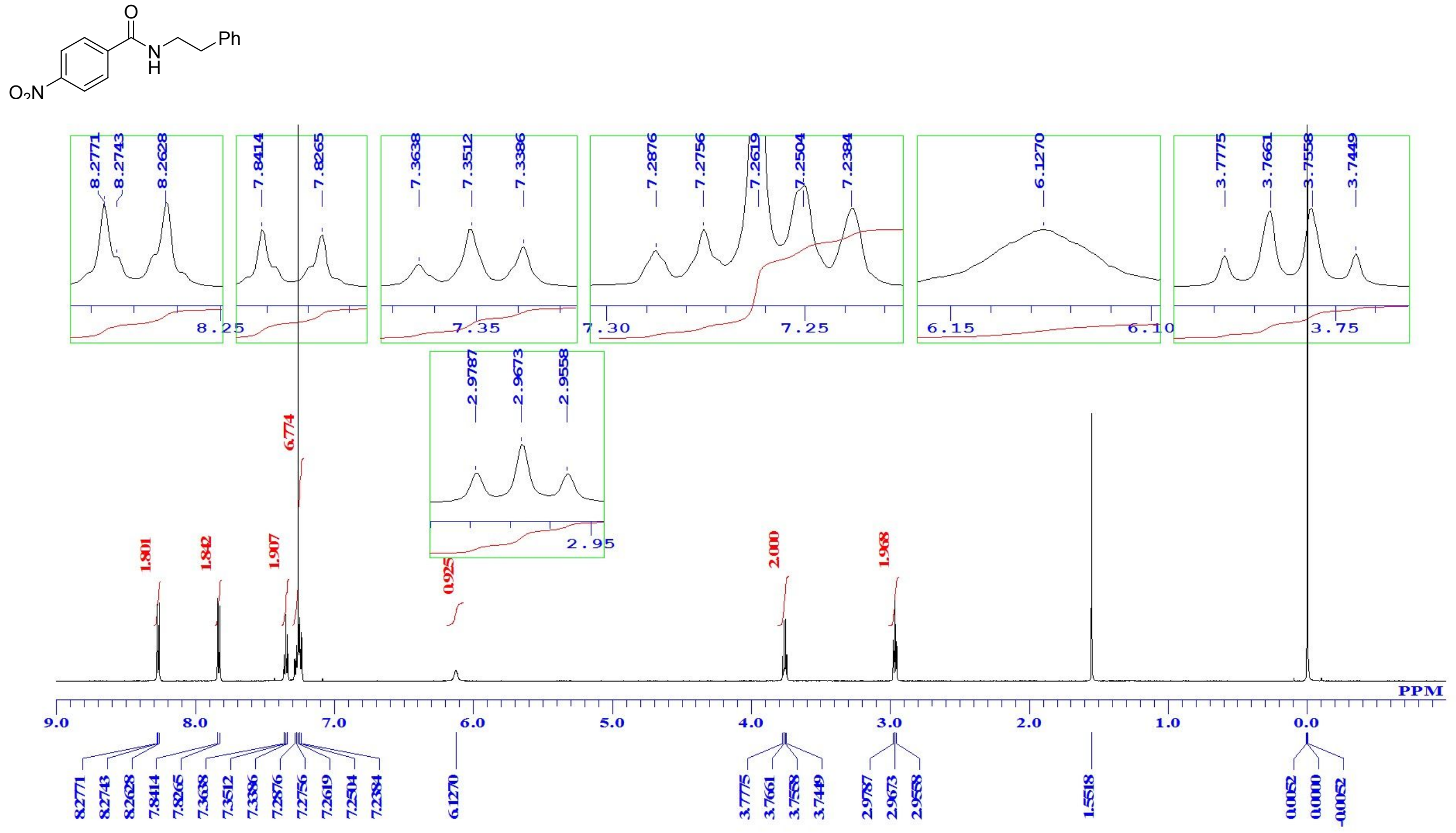


${ }^{1}$ H NMR: $N, N$-Diethyl-benzamide (17cb)
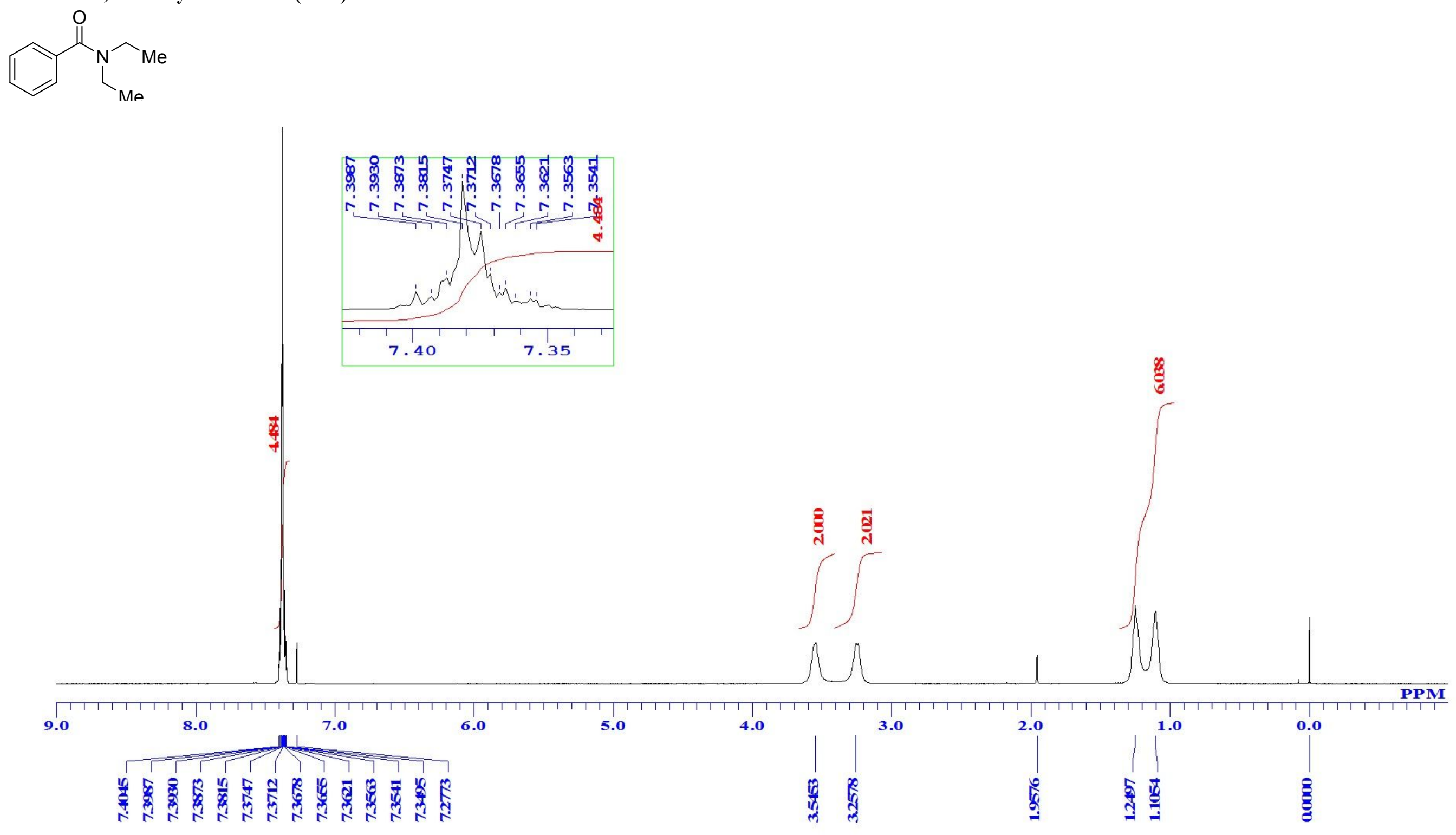
${ }^{1}$ H NMR: $N, N$-Dibenzyl-benzamide (17cc)

$\overbrace{P h}^{O} \sim_{P h}^{O N}$

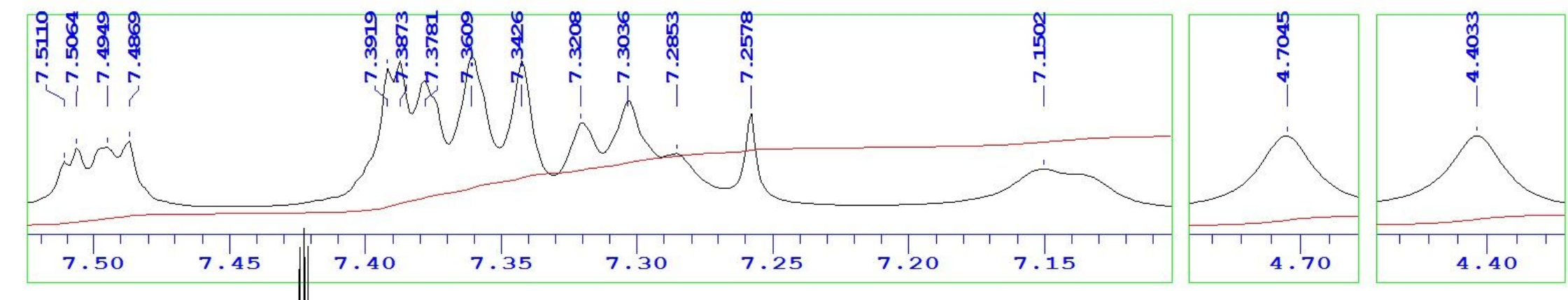

$\stackrel{6}{7}$
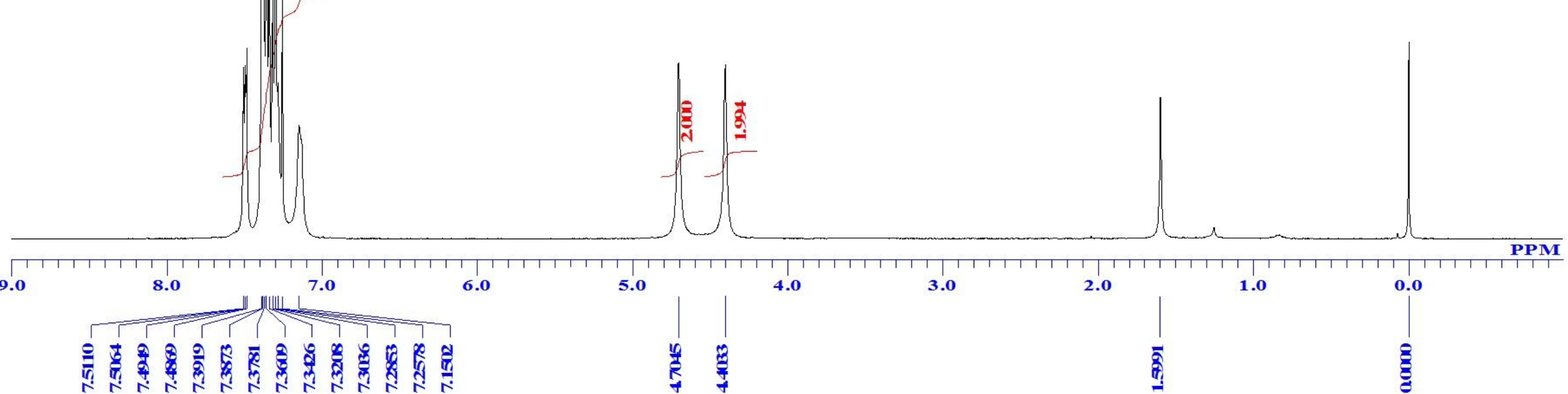
Methyl ((benzyloxy)carbonyl)-L-phenylalanyl-L-alaninate (17fd)

$\mathrm{CbzHN} \underbrace{\bigcup_{\mathrm{H}}^{\mathrm{O}}}_{\overline{\mathrm{B} n}} \overbrace{\mathrm{O}}^{\mathrm{Me}} \mathrm{OMe}$
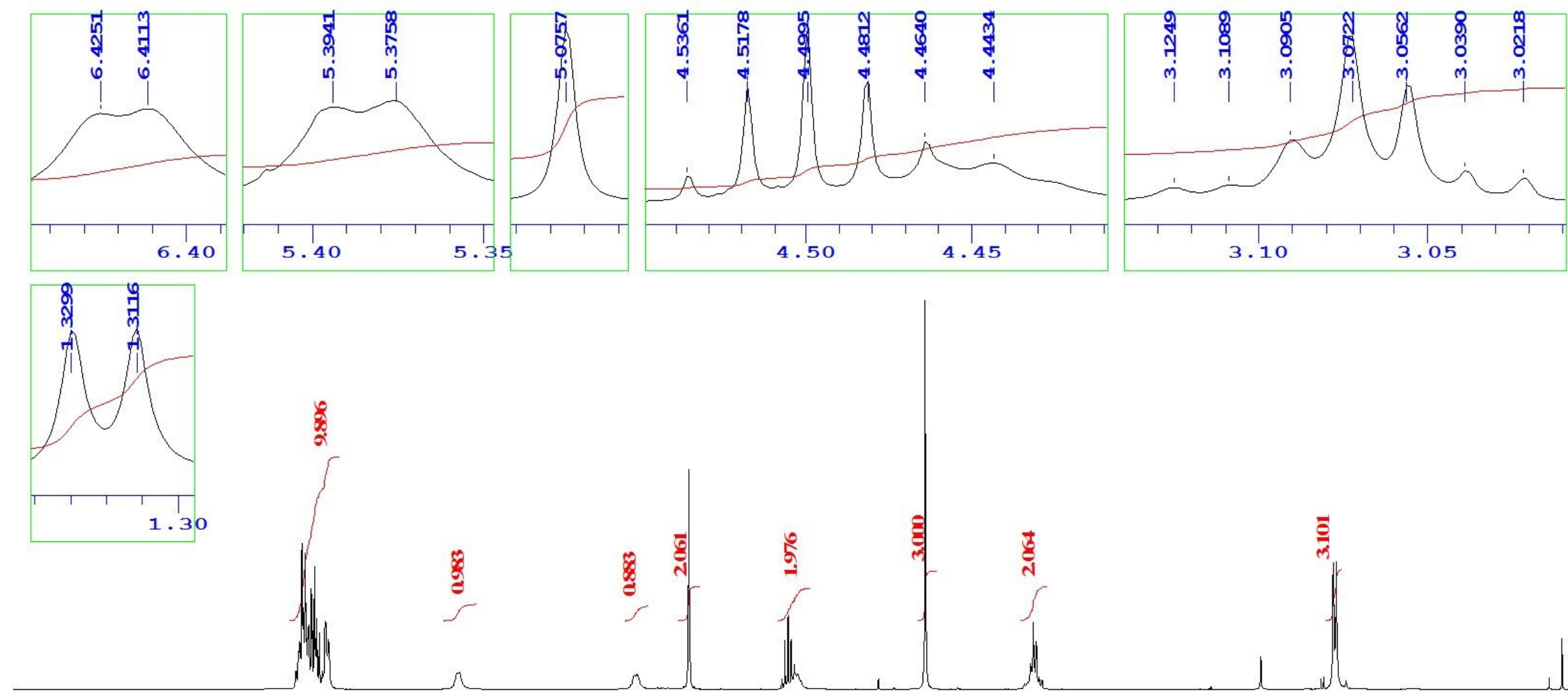

$$
\text { . }
$$

3.10

3.05

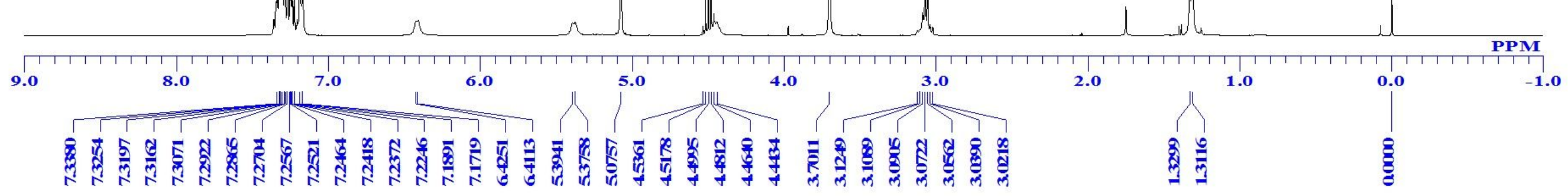


Methyl ((benzyloxy)carbonyl)-D,L-phenylalanyl-L-alaninate (D,L-17fd)

$\mathrm{CbzHN} \underbrace{\mathrm{Me}}_{\mathrm{Bn}} \mathrm{OMe}$
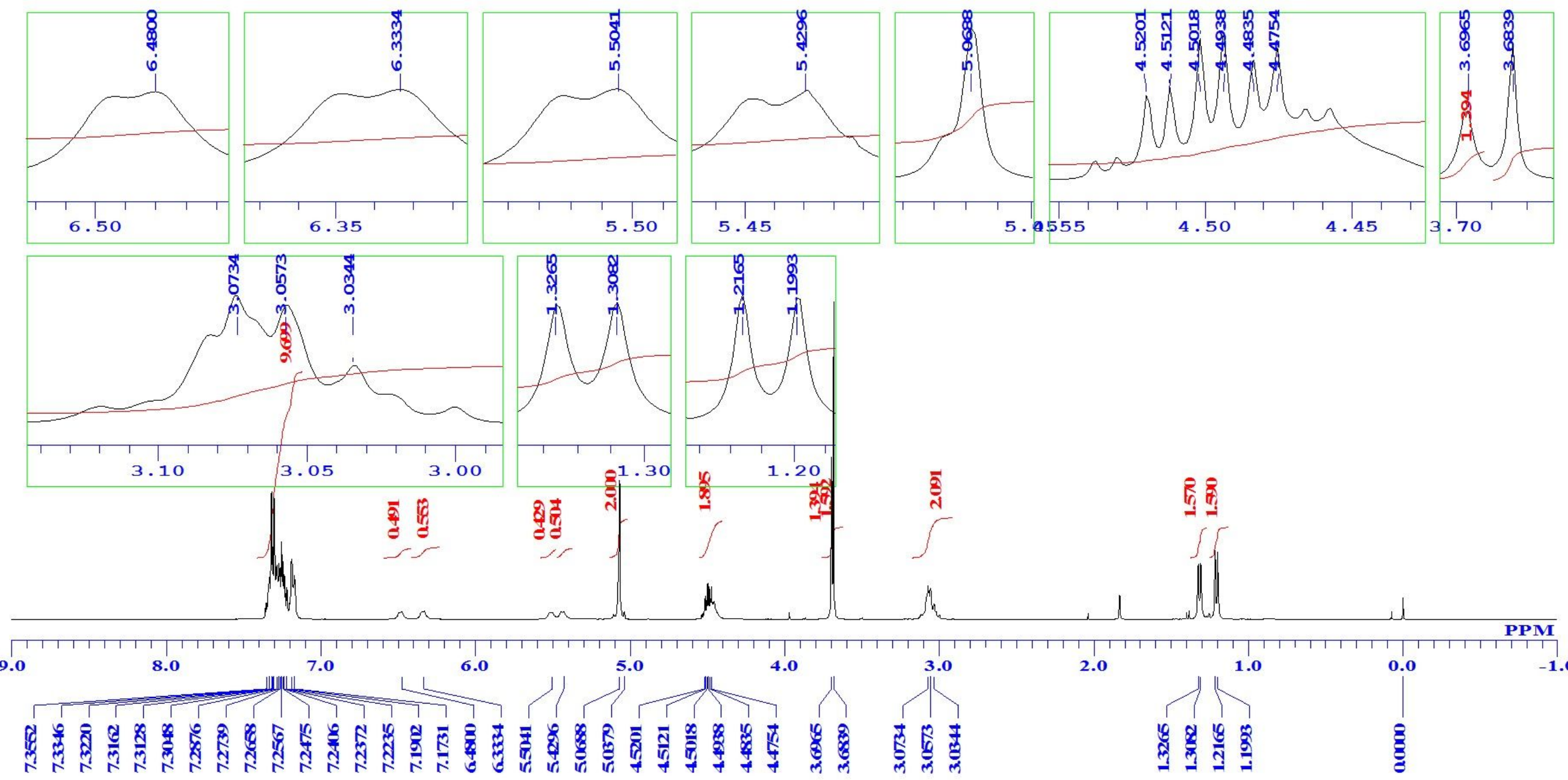

3.00

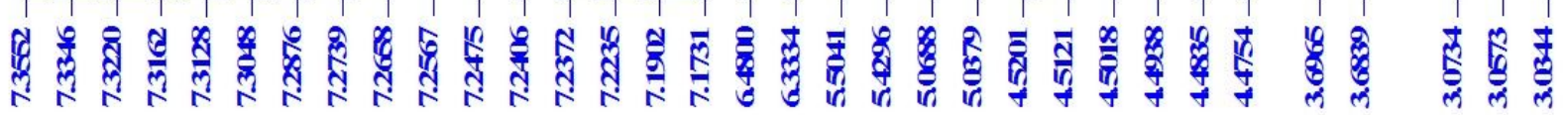

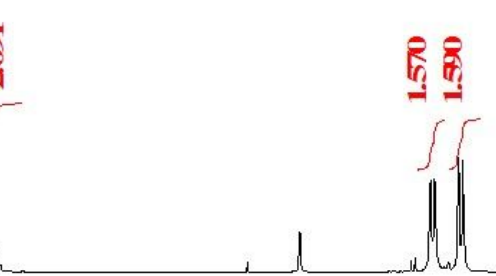

PPM

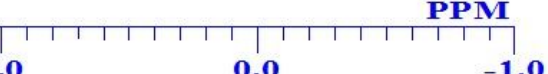

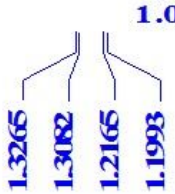

